\title{
Expandable Polymer Assisted Wearable Personalized Medicinal Platform
}

Thesis by

Wedyan Babatain

In Partial Fulfillment of the Requirements

For the Degree of

Master of Science

King Abdullah University of Science and Technology

Thuwal, Kingdom of Saudi Arabia

April, 2019 


\section{EXAMINATION COMMITTEE PAGE}

The thesis of Wedyan Babatain is approved by the examination committee.

Committee Chairperson: Dr. Muhammad Mustafa Hussain

Committee Member: Dr. Mohamed Slim Alouini

Committee Member: Dr. Udo Schwingenschloegl 
(C) April, 2019

Wedyan Babatain

All Rights Reserved 


\section{ABSTRACT \\ Expandable Polymer Assisted Wearable Personalized Medicinal Platform \\ Wedyan Babatain}

Conventional healthcare and the practice of medicine largely relies on the ineffective concept of one size fits all. Personalized medicine is an emerging therapeutic approach that aims to develop an advanced therapeutic technique that provides tailor-made therapy based on every individuals' needs by delivering the right drug at the right time with the right amount of dosage. The advancement in technologies such as flexible electronics, microfluidics, biosensors, and advanced artificial intelligence can enable the realization of a truly effective personalized therapy. However, currently, there is a lack for a personalized minimally-invasive wearable closed-loop drug delivery system that is continuous, automated, conformal to the skin and cost-effective. Thus, this thesis focuses on the design, fabrication, optimization, and application of an automated personalized microfluidics drug delivery platform augmented with flexible biosensors, heaters, and expandable polymeric actuator. The platform provides precise drug delivery with spatiotemporal control over the administered dose as a response to real-time physiological changes of the individual. The system is flexible enough to be conformal to the skin and drug is transdermally administered through biocompatible microneedles. The platform includes a flexible multi-reservoir microfluidics layer, flexible and conformal heating elements, skin sensors and processing units which are powered by a lightweight 
battery integrated into the platform. The developed platform was fabricated using rapid, cost-effective techniques that are independent of advanced microfabrication facilities to expand its applications to low-resource setting and environments. 


\section{ACKNOWLEDGEMENTS}

There are so many people that I would like to thank whom without their guidance, support, and encouragement, this thesis project would not have been completed. First, I would like to thank my advisor professor Muhammad Mustafa Hussain for his continuous guidance throughout this project and my journey here at KAUST. His mentorship has been instrumental to my growth as an aspiring independent scientist and researcher. I would also like to extend my appreciation to professor Mohamed Slim Alouini and professor Udo Schwingenschloegl for taking the time to serve as members in my thesis committee. I am thankful for members of core labs like Mr. Ulrich Büttner, who has always provided me with insightful feedback to curate my ideas ever since I came to KAUST as an intern and trained me to work in the microfluidics core lab. I would also like to thank Melvin Cruz and all the members of the $\mathrm{MMH}$ labs and all my friends here at KAUST.

I would like to express my deep appreciation to my amazing parents, Hassan and Sameerah Babatain for their unconditional love and support throughout my work in this thesis and throughout my life. Their constant encouragement has always been the

driving force for my development. My appreciation is extended to my sisters and brothers who are the best support system I could ever ask for. 
Finally, I would like to dedicate this thesis to my beloved grandmother, who has passed away recently, may Allah bless her soul. She has always encouraged me to continue my studies and to relentlessly chase my dreams. 
TABLE OF CONTENTS

Page

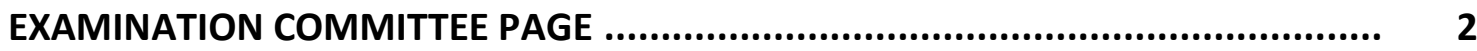

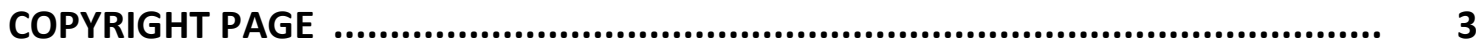

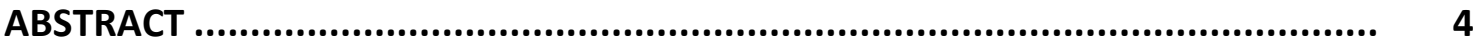

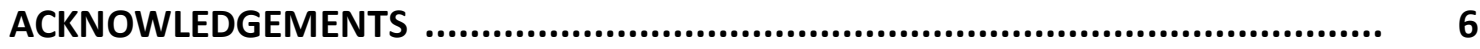

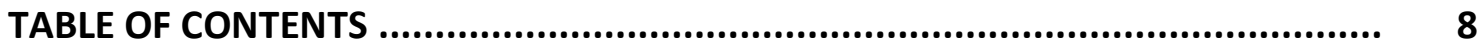

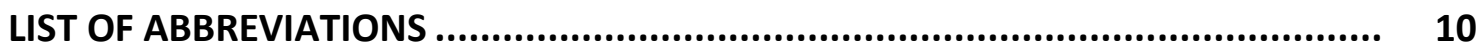

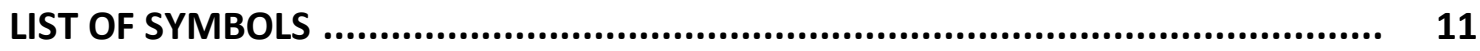

LIST OF ILLUSTRATIONS ......................................................................... 13

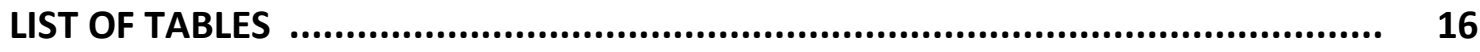

Chapter 1: Introduction and Background.......................................................... 16

1.1 Personalized Medicine .............................................................................. 16

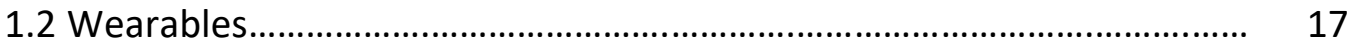

1.2.1 Wearable Sensors ............................................................... 19

1.2.2 Wearable Transdermal Drug Delivery Devices........................ 26

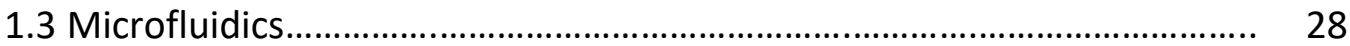

1.3.1 Mixing in Microfluidic Systems............................................. 29

1.4 Closed-loop Transdermal Drug Delivery Systems..................................... 30

1.4.1 Requirements for Transdermal Drug Delivery systems ......... 32

1.5 Research Objectives and Outline ............................................................ 33

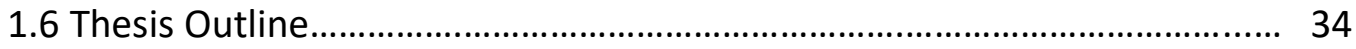

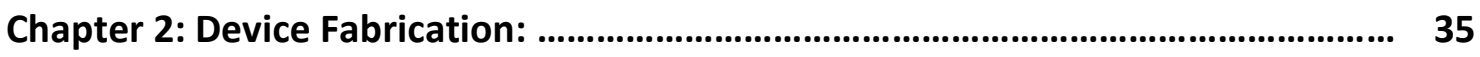

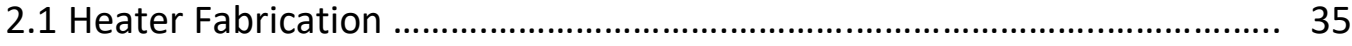

2.1.1 Microheater Array ................................................................ 36

2.1.2 Operating Principle of the heaters ......................................... 36

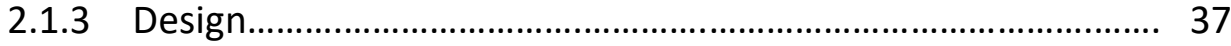

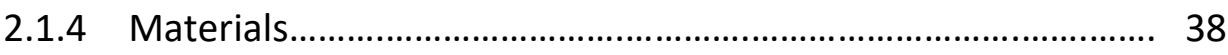

2.1.5 Fabrication Process ........................................................ 39

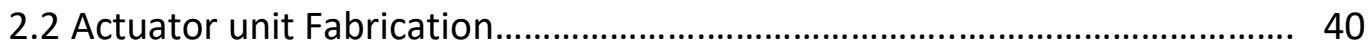

2.2.1 Expancel Microspheres ............................................................. 40

2.2.2 Expancel-PDMS composite .................................................. 42 
2.2.3 Operating Principle of the expancel as an actuator.................. 42

2.2.4 Fabrication Process .................................................................... 43

2.3 Microfluidics Layer Fabrication.................................................................... 44

2.3.1 Design \& Layout....................................................................... 44

2.3.2 Micromixer Geometry............................................................ 46

2.3.3 Reservoir Geometry............................................................. 49

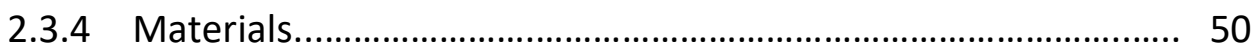

2.3.5 Fabrication Process.................................................................. 50

2.4 Wearable Sensors Fabrication................................................................... 52

2.4.1 Operating principle of Temperature \& Humidity Sensors.......... 52

2.4.2 Design......................................................................................... 54

2.4.3 Materials ................................................................................. 54

2.4.4 Fabrication Process.................................................................. 55

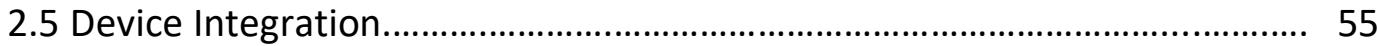

2.5.1 Bonding Process....................................................................... 55

2.5.2 Microneedles integration..................................................... 57

2.5.3 Device disposability \& drug re-fill............................................ 58

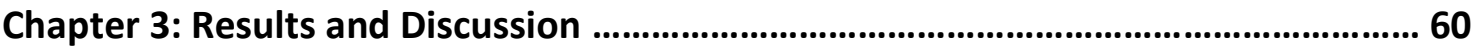

3.1 Heater Characterization \& Optimization ................................................. 60

3.1.1 Heater Geometry Optimization............................................. 60

3.1.2 Heater Characterization ........................................................ 63

3.2 Actuator Characterization \& Optimization................................................. 64

3.2.1 Expancel-PDMS layer Thickness optimization........................... 66

3.3 Microfluidics Layer Characterization \& Optimization................................ 69

3.3.1 Reservoir Geometry Optimization............................................. 69

3.3.2 Microfluidics Mixing............................................................... 70

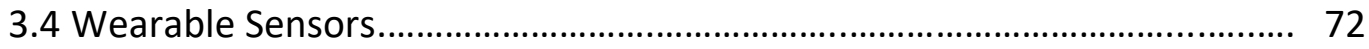

3.4.1 Temperature and Humidity Sensors Characterization.............. 72

3.5 System Integration........................................................................... 74

3.5.1 Closed-Loop Experiment........................................................ 77

Chapter 4: Concluding Remarks and Future Work .................................................. 78

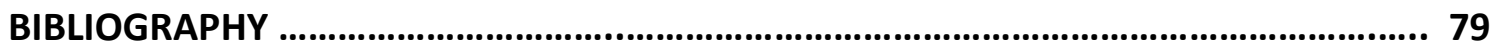




\section{LIST OF ABBREVIATIONS}

$\begin{array}{ll}\text { CAD } & \text { Computer-aided design } \\ \text { IR } & \text { Infrared radiation } \\ \text { LOC } & \text { Lab on chip } \\ \text { MEMS } & \text { Microelectromechanical systems } \\ \text { NTC } & \text { Negative temperature coefficient } \\ \text { PDMS } & \text { Polydimethylsiloxane } \\ \text { PI } & \text { Polyimide } \\ \text { PTC } & \text { Positive temperature coefficient } \\ \text { TCR } & \text { Temperature coefficient of resistance } \\ \text { RFID } & \text { Radio-frequency identification } \\ \text { SEM } & \text { Scanning electron microscope } \\ \text { TCR } & \text { Temperature coefficient of resistance } \\ \text { ( } \mu \text { TAS) } & \text { Micro totally analysis systems }\end{array}$




\section{LIST OF SYMBOLS}

\begin{tabular}{|c|c|}
\hline$A$ & Area \\
\hline C & Capacitance \\
\hline${ }^{\circ} \mathrm{C}$ & Degree Celsius \\
\hline$D$ & Diffusion constant \\
\hline$d$ & Distance \\
\hline$j$ & Diffusion flux \\
\hline$I$ & Current \\
\hline$k$ & Boltzmann constant \\
\hline$L$ & Length \\
\hline$P$ & Power \\
\hline$Q$ & Volumetric flow rate \\
\hline$r$ & Radius \\
\hline$R$ & Resistance \\
\hline$R e$ & Reynolds Number \\
\hline$T$ & Temperature \\
\hline$t$ & Time \\
\hline$v$ & Velocity \\
\hline$V$ & Voltage \\
\hline$W$ & Width \\
\hline$x$ & Position \\
\hline
\end{tabular}




$\begin{array}{ll}\Delta \mathrm{T} & \text { Temperature difference } \\ \Delta \mathrm{R} & \text { Resistance difference } \\ \varepsilon_{0} & \text { Permittivity of vacuum } \\ \varepsilon_{r} & \text { Relative permittivity of the dielectric material } \\ \eta & \text { A mathematical constant defined as the ratio of the circle's } \\ \pi & \text { Density } \\ \text { circumference to its diameter } \\ \rho\end{array}$




\section{LIST OF ILLUSTRATIONS}

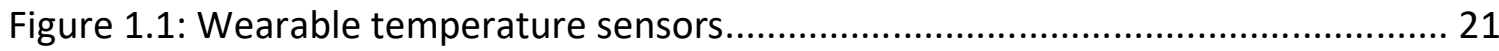

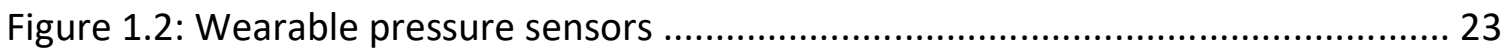

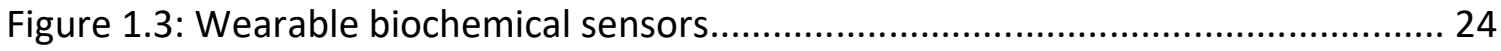

Figure 1.4: Wearable multisensory platform.......................................................... 25

Figure 1.5: Wearable paper-based multisensory platform....................................... 25

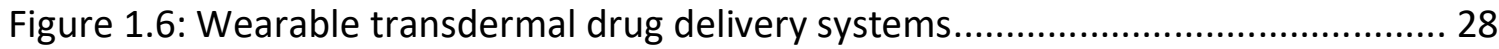

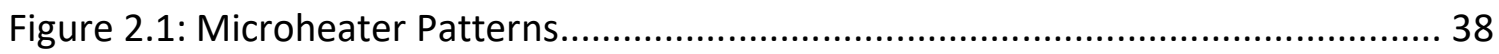

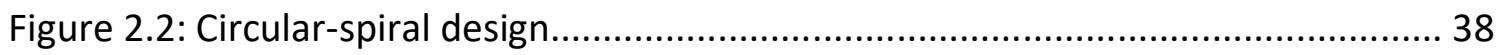

Figure 2.3: Fabrication process of the microheaters layer..................................... 40

Figure 2.4: Schematic illustration of expancel expansion principle................................ 41

Figure 2.5: Schematic illustration of the operation principle behind of the device........ 43

Figure 2.6: Optical photograph of fabricated expandable composite.......................... 44

Figure 2.7: Schematic of design 1 of the microfluidics reservoir layer.......................... 45

Figure 2.8: Schematic of design 2 of the microfluidics reservoir layer......................... 45

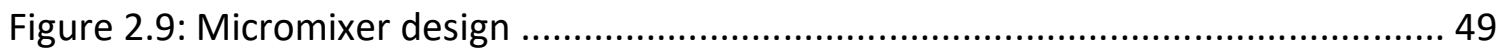

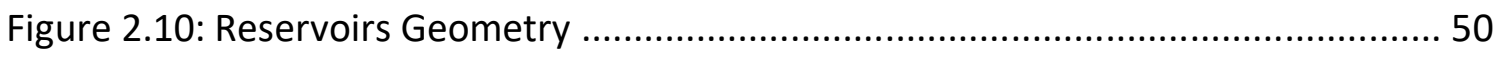

Figure 2.11: Fabrication process of the microfluidics reservoir layer.......................... 52

Figure 2.12: Optical photograph of the 3D printed master mold................................. 52

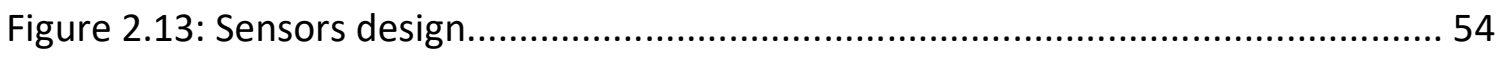

Figure 2.14: Exploded view schematic illustration of device...................................... 56

Figure 2.15: Optical photograph of Micropoint stainless-steel microneedle array ....... 57

Figure 2.16: Schematic of microneedle integration............................................... 58

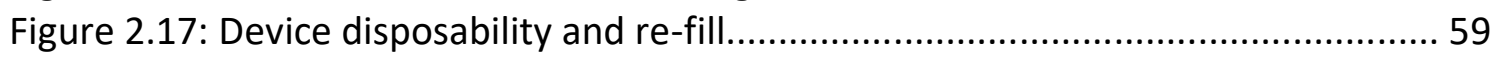

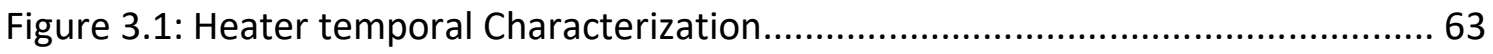

Figure 3.2: Thermal characterization of microheaters............................................ 64

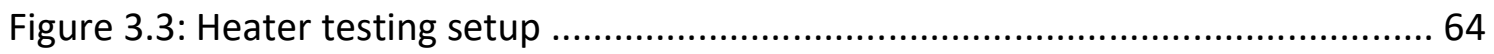

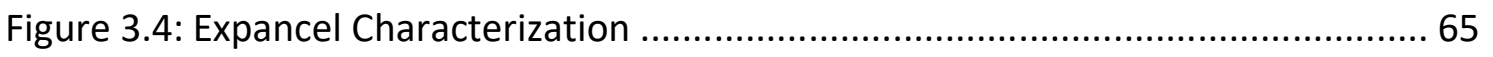

Figure 3.5: SEM images of Expancel ${ }^{\circledR}$ microspheres.................................................. 65

Figure 3.6: Drug infusion volume as a function of thickness variation of expancel composite.................................................................................................... 66

Figure 3.7: Drug infusion volume versus expancel composite thickness showing the two

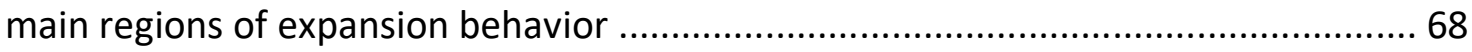

Figure 3.8: Drug infusion volume as function of the reservoir shape/geometry......... 70

Figure 3.9: Reservoir Geometry Conformity....................................................... 70

Figure 3.10: Validation of microfluidic mixing capability of the device....................... 71

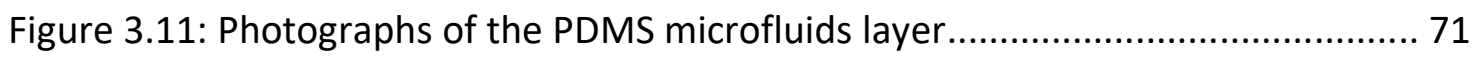

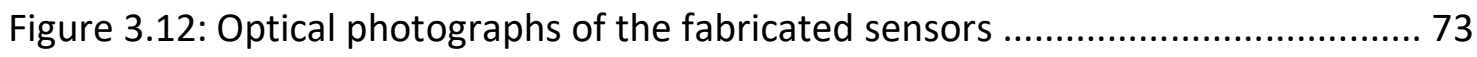

Figure 3.13: Temperature sensor characterization plot ...........................................73

Figure 3.14: Humidity sensor characterization plot ................................................ 74

Figure 3.15: Microneedle integration and testing ........................................... 75 
Figure 3.16: Photographs of the integrated system......................................... 76

Figure 3.17: Photograph of the wearable platform on a wrist .............................. 76

Figure 3.18: Disposability of the wearable platform.......................................... 76

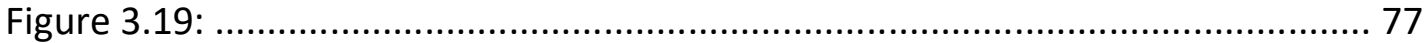




\section{LIST OF TABLES}

Table 3.1 Thermal Analysis of microheater patterns ......................................... 28 


\section{Chapter 1: Introduction and Background}

\subsection{Personalized Medicine}

Conventional healthcare and the practice of medicine largely relies on the concept of one size fits all. This model offers the same therapeutic approach to treat a large group of people with a similar medical condition or disease. The human body is one of the most variable systems that exists, so the probability that the treatment will be effective for all patients is extremely low. Additionally, some individuals might experience adverse effect as a result of using a generally prescribed drug. Personalized medicine is a therapeutic approach that has been growing in the past few years. The aim of personalized medicine is to develop advanced therapeutic techniques by delivering the right drug at the right time using the right amount of dosage. Personalized therapy is considered superior to conventional therapy because it can provide tailor-made medications based on an individuals' daily physiological changes $\mathrm{m}$ biomarkers, genetics, and phenotypic attributes $^{[1]}$. The recent technological advancement in wearables, sensors and microfluidics devices enable the realization of precision medicine and its advantages. These advancements are not only limited to sensors and wearables technologies, but also big data, machine learning, artificial intelligence imaging, and gene sequencing all are contributing to the realization of personalized healthcare. These technological advancements act as accelerators and drivers to modern's day precision medicine. Personalized medicine not only increases the safety of therapeutics by minimizing side effects, but it also decreases the cost associated with therapy by eliminating trials and 
errors of multiple treatment types and modalities and finding the one that maximizes the therapeutic output. These technologies provide ample amount and wide range of valuable medical information about each patient. Although initially creating such devices was solely for health monitoring purposes, the medical significance of such information will be maximized if they are actionable. In other word, when this information is being used to deliver or treat the diseased tissue with the appropriate type of medication. Only when that is achieved, technological disruption can be made in the field of personalized medicine.

\subsection{Wearables}

An active goal of many researchers working on the field of wearables is to better understand the complex human body by capturing electrophysical and electrochemical signals that have medical significance. To provide better preventative medical and therapeutic techniques, we need to be able to capture all possible signals and critical parameters from the human body. The goal is to gain valuable information about the human body and the implications of such physiological signals to provide better health monitoring and therapeutic techniques. The most widespread conventional health monitoring systems that provide such capabilities include bulky rigid electronics that are wired to sensors and fixed around the tissue of interest using straps. The use of such systems also requires the existence of advanced laboratory facilities and the presence of professional personnel to obtain such signals. Although these well-established systems can provide quite accurate reading and measurements of the major body vital signals, the 
mechanical mismatch between the human body tissue and the rigid electronics as well as the need for technical personnel to operate devices are some of the challenges that limit the widespread deployment of such health mentoring systems to a wider population. Due to the previously mentioned reasons, the field of flexible wearable electronic systems has emerged in the past few years. Several groups including ours are working extensively on developing compliant, flexible electronics that can be used as a foundation to develop bio integrated sensors and actuators that provide seamless and intimate contact with the human body while enabling various complex functionalities. The realization of such devices can be attributed due to the ongoing quest of miniaturization of integrated circuits using Moore's law and the constant adoption of new emerging materials and techniques ${ }^{[2]}$. Although these devices have some challenges related to its accuracy and reliability, the market of wearable electronics is expected to reach around $\$ 25.19$ Billion by 2020 which shows the volume by which this field is growing ${ }^{[3]}$. This projected forecast will drive researchers to continue the development of miniaturized flexible and wearable electronics that offer excellent accuracy and reliability levels and can overcome the main limitation of conventional bulky planar electronics. Thus, it is imperative to establish a reliable and intimate contact with the skin to obtain more accurate signals and more meaningful readings. Not only is the design of such devices is critical, but also the materials being used as a substrate for these devices is another important factor to overcome the limitation of conventional rigid electronics. A common approach is implemented by thinning down silicon which achieves excellent bending capabilities. Such devices have the ability to not only revolutionize the medical diagnostics field through 
sensors but also the medical treatment field via actuating drug delivery devices. This section will introduce the state of the art and recently developed wearable biomedical devices including wearable biosensor for health monitoring and wearable drug delivery devices for therapeutics.

\subsubsection{Wearable Sensors}

Epidermal biosensors that are mounted on the skin can be grouped into three main categories based on the nature of the signal being captured by the sensor: Electrophysical, electrochemical or environmental sensors. Skin biosensors can be used to measure biophysical signals which can be translated into physiological indications about various conditions of the body such as the activity of heart, muscles, and brain. This signal can be a change in temperature, humidity, respiration, pressure, strain, biopotential and motion. The sensing mechanisms used in these sensors can be resistivebased, capacitive-based or it can utilize piezoelectric properties. Conventional electronics used in lab settings have many issues related to skin irritations and comfortability. That is because such electronics use hard-wired connections, adhesive gels and wraps in order to be able to capture the desired signal. A considerable number of advances have been made in material sciences and design of thin electrodes on flexible substrates. These electrodes form the basic structure for the majority of sensing elements and offer seamless and conformal contact to the skin for more accurate continues readings ${ }^{[4]}$. 
Temperature is one of the most important vital signs of the human body. An elevation or change in the body's temperature from the normal range can be an indication of the presence of different types of undesired physiological changes such as inflammation, infection, and diseases ${ }^{[5][6][7][8]}$. This emphasizes the increased need for flexible sensory devices that can conformably adhere to the skin and contentiously monitor the human body's temperature with a high level of accuracy. The most common sensing mechanism used for temperature sensors is based on the thermosensitive effect. Metals and semiconducting materials experience increase in its resistance when its temperature is increased due to the positive temperature coefficient (PTC) or negative temperature coefficient (NTC) of the resistance ${ }^{[9][6]}$.Carbon nanotubes ${ }^{[10][11][12]}$ and graphene ${ }^{[13]}$ [9][14][15][16][17] are two of the mostly used materials for resistive based temperature wearable sensors. Yang et al. reported a flexible ultrasensitive wearable temperature sensor based on graphene nanowalls. The developed sensor exhibits high stability and sensitivity which makes it a candidate for real-time measurement that can be used for personalized healthcare in the future. The sensors are shown in figure1.1a and 1.1b ${ }^{[17]}$. Another NTC type resistive temperature sensor is reported by Yan et al. which utilizes graphene on a flexible substrate to form a thermistor with tunable thermal index. The stretchability of the substrate caused an increase in sensitivity at high strain conditions as the strain increased the resistance of the contact gold nanowire electrodes ${ }^{[9]}$. It is quite important to be able to measure minor changes in the body's temperature over a short time with high resolution as it is an important health biomarker. Other widely used materials for temperature sensing elements are gold ${ }^{[18][19]}$, liquid metals, conducting 
polymers $^{[20][21][22]}$, silk ${ }^{[23]}$ and hydrogels ${ }^{[24]}$. An example of a temperature sensor based on gold electrodes can be seen in figure 1.1e and 1.1f This sensor exhibits a fast response time to temperature variations as the gold has the properties of low thermal mass. The sensor utilizes a filamentary serpentine structure in an open mesh interconnect arrangement. The sensor is ultrathin and stretchable to enable conformal lamination onto the skin and provide non-invasive spatial mapping of the temperature of the skin [25] Another mechanism of temperature sensing was developed by some groups using thermochromic materials that respond to change in color upon a change in temperature. Gao et al. developed a photonic epidermal sensor shown in figure $1.1 \mathrm{~g}$ and figure $1.1 \mathrm{~h}$ that utilizes thermochromic liquid crystals embedded into PDMS substrate. PDMS add elastic properties for the sensor

for seamless skin application. Camera and computational algorithms are used for precise colorimetric temperature detection ${ }^{[26]}$.

(a)

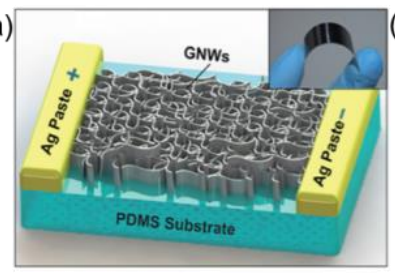

(c)

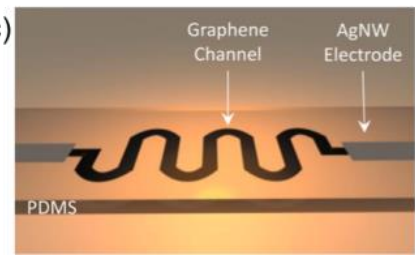

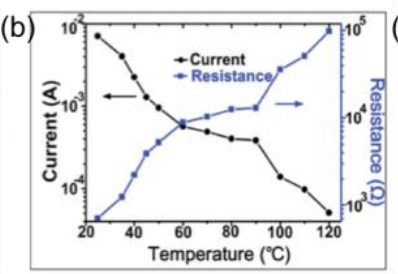

(d)

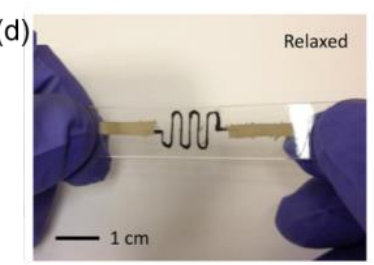

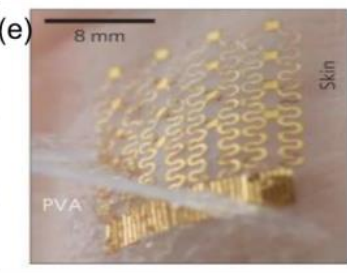

$(\mathrm{g}$

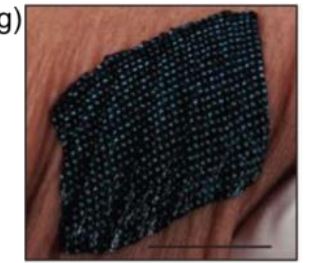

(f)

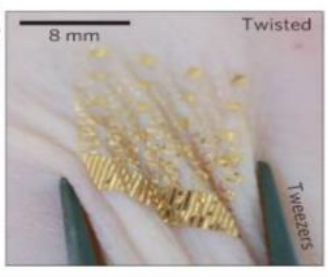

(h)

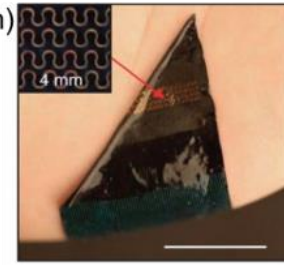

Figure 1.1: Wearable temperature sensors a) Schematic demonstration of the structure of graphene temperature sensor on PDMS substrate. b) Characterization of the sensor shown in a showing the current and resistance versus temperature graphs when $5 \mathrm{~V}$ voltage is applied. (c,d) schematic demonstration and optical images of graphene thermistor under relaxed state. (e,f) TCR ultra-think temperature sensor based on gold-electrode mesh showing its high compliance when applied into skin under pinching and twisting conditions. (g,h) Optical images of thermochromic liquid crystals temperature sensor showing its deformation on skin as the communication unit of as RF serpentine antenna. 
Another type of important skin sensor is pressure sensors. Pressure sensors enable the monitoring of vital human signals such as blood pulse, heart rate, and respiration rate. Some pressure sensors are resistive-based which rely on a change of material resistivity, and other are capacitive based which rely on changes in the properties of the dielectric material used ${ }^{[27]}$. An example of a resistive-based wearable pressure sensor was reported by Gong et al. ${ }^{[28]}$. The sensor is made out of ultrathin gold nanowire sandwiched between twos PDMS layer as seen in figure 1.2a and it exhibits high sensitivity pressure. The sensor, although ultrathin, has shown stable performance under the influence of mechanical deformations such as bending, pressing and twisting ${ }^{[28]}$.

An example of capacitive-based pressure sensor with a novel micro hair structure was reported by Pang and others ${ }^{[29]}$. The integration of an array of micropillars improves the interface with the skin and the sensor sensitivity as it expands the contact area between the sensor and the irregularities areas of the skin. Figure $1.2 \mathrm{f}$ shows a characterizations graph of the capacitive pressure when used for monitoring the pulse produced by the radial artery of the wrist. 
(a)

(b)

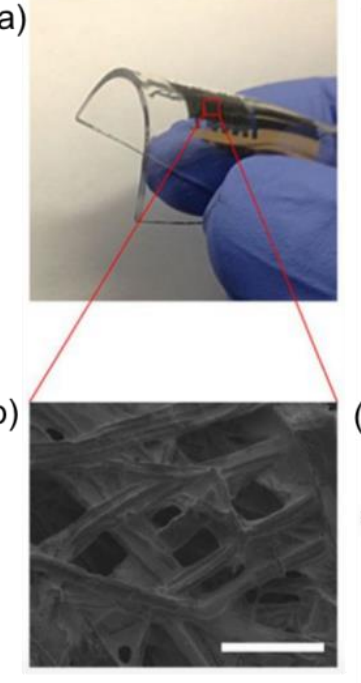

(c)
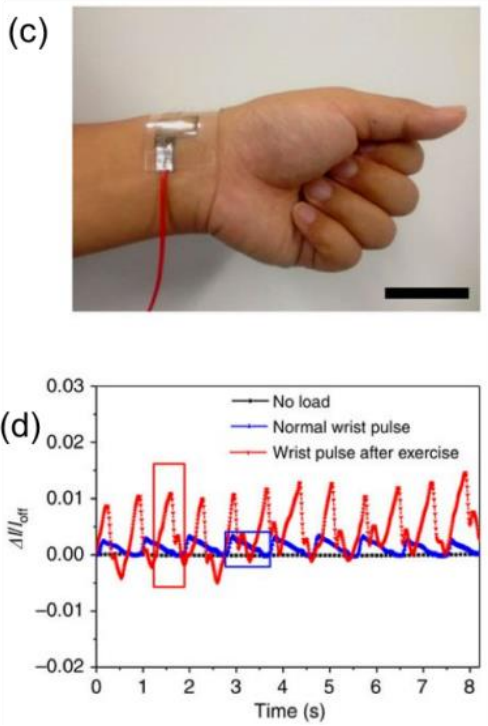

(e)

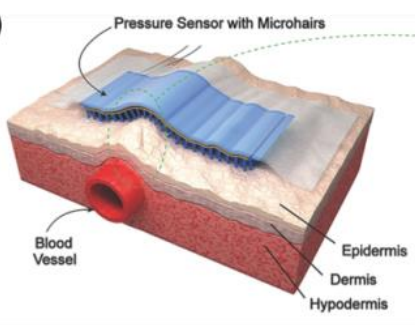

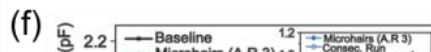

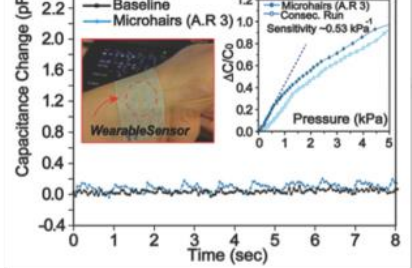

Figure 1.2. Wearable pressure sensors a) Optical image of the gold nanowire ultrathin pressure sensor showing its flexible capability. b) SEM image of the surface of the sensor with gold nanowires. (c, d) images and graph showing the sensor performance when applied on the wrist to monitor the heart beat under normal and exercise states. e) Schematic depiction of capacitive-based microhair wearable pressure sensor f) characterization graph of the sensor when used to monitor the pulse wave of the radial artery from the wrist.

In order to capture more accurate information about the health status of the human body, biochemical signals from targets like metabolites and electrolytes need to be measured in addition to the previously mentioned biophysical signals. As conventional methods of biochemical signals monitoring rely on bulky laboratory analyzers and skilled personnel, it limits its use in settings outside laboratory environments. For electrochemical signals, epidermal interface plays such an important factor for capturing signals from invasive biofluids like the blood or noninvasive biofluids such as sweat and saliva without causing any irritations. An example is a metabolic multi-sensing platform that was developed by Javey et al. shown in figure 1.3a and 1.3b. The device is consisting of gold electrodes patterned on a flexible PET substrate. It contains sensing elements to capture the concentration of lactate, glucose in the sweat. The device is flexible enough to conform to different parts of the body and can be used for personalized diagnostics 
applications $^{[30]}$. Another sensor was fabricated using layers of carbon nanotubes with gold electrodes on a stretchable substrate. The sensor is capable of detecting glucose and $\mathrm{pH}$ levels in the sweat with sensitivities of $71.44 \mathrm{mV} \mathrm{pH}-1$ for $\mathrm{pH}$ and $10.89 \mu \mathrm{AmM}-1 \mathrm{~cm}-2$ and for glucose ${ }^{[31]}$. One of the challenging limitations for flexible wearable sensors is the development of ultrathin, flexible communication units. A recent electrolyte sensor developed by Rose et al., features an adhesive radio-frequency identification (RFID) as the component of communication which resulted in lightweight, battery-free sodium sensor with excellent epidermal interface ${ }^{[32]}$.

(a)

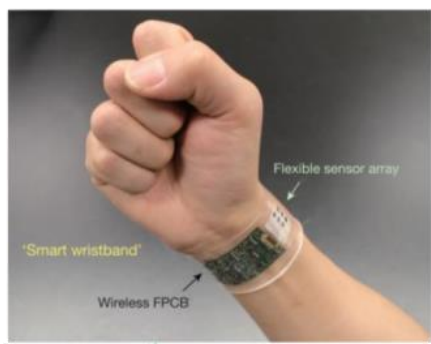

(b)

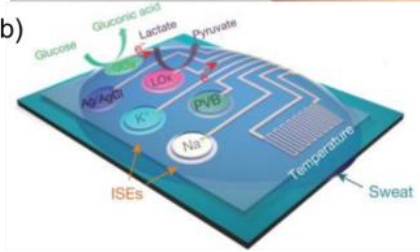

(c)

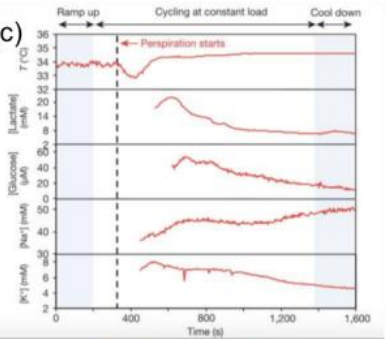

(d)

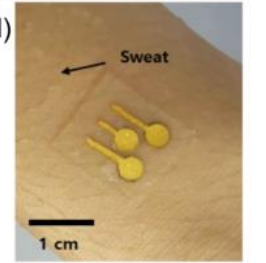

(e)
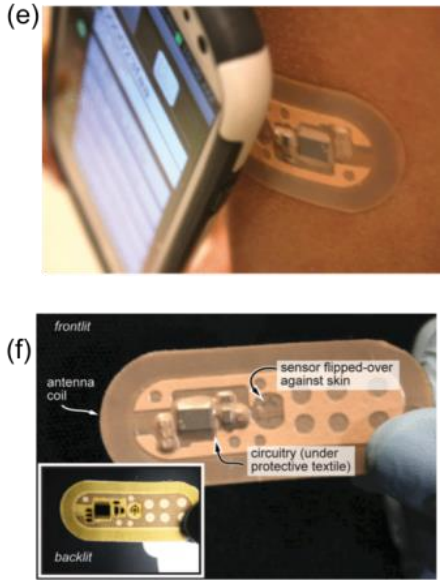

Figure 1.3. Wearable biochemical sensors: a) Optical image of the wearable flexible integrated mutli-sensory platform when applied on the wrist including wireless PCB unit. b) Schematic representation of the different sensing elements for lactate glucose and electrolytes. c) The performance of the sensor in real-time collected from analytes in the sweat during indoor cycling experiment. d) Optical of a stretchable gold nanosheets electrodes of glucose sweat sensor conformed to the skin. e,f) Optical image of stretchable sodium sweat sensor with battery free data collection radio-frequency identification unit.

Recent effort by our group reported the development of a multi-sensory wearable platform where the platform is composed of a stack of multiple type of sensors for various vital signals to provide comprehensive real-time health monitoring ${ }^{[33][4]}$. The platform has the ability to simultaneously detect signals such as humidity, temperature $\mathrm{pH}$, pressure, touch, flow, motion, and proximity. 
(a)

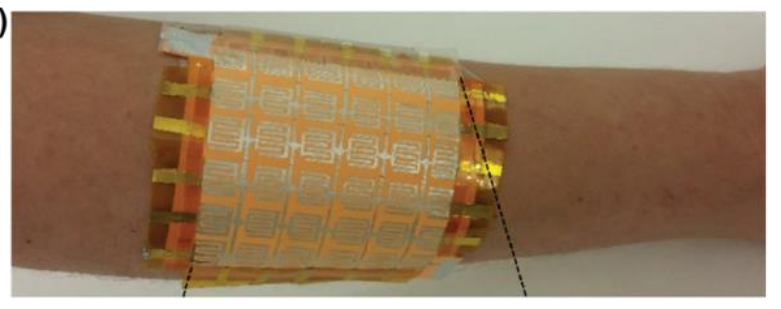

(b)

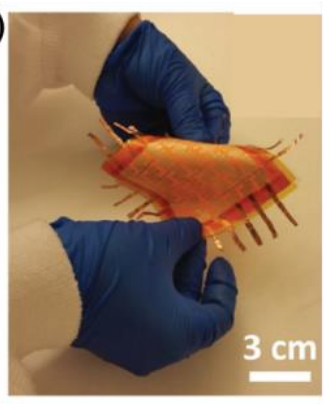

(c)

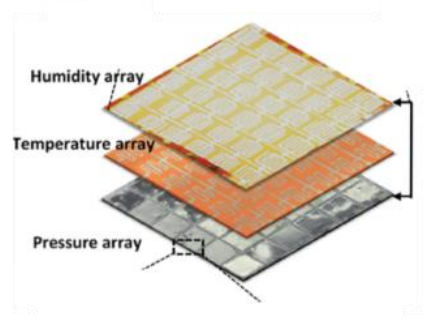

(d)
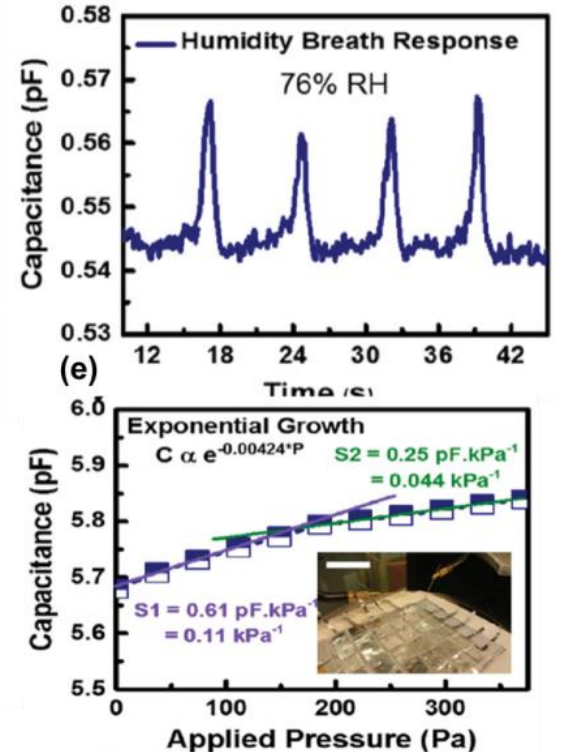

Figure 1.4. Wearable multisensory platform for simultaneous monitoring. a) Optical photograph of a flexible "paper skin" applied around the arm. b) 3D demonstration of the paper skin stack consisting of humidity, temperature and pressure arrays. c) Optical image of the of the senor array deformed. d) Characterization of the humidity sensor showing real-time response to humidity levels when cycles of human breath are applied. e) Characterization of the pressure.

(a)

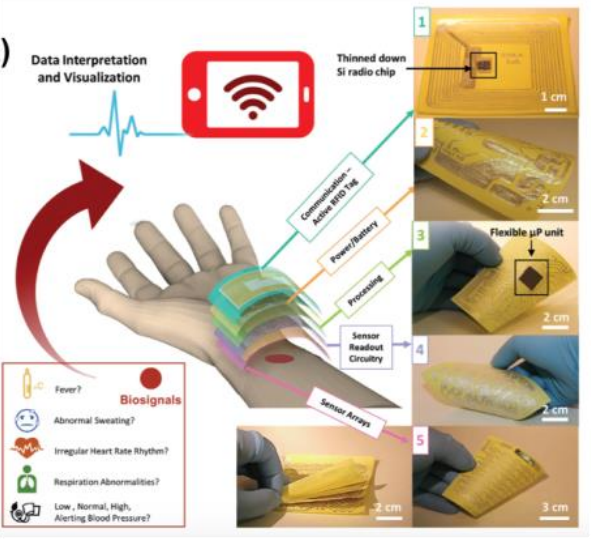

(b)

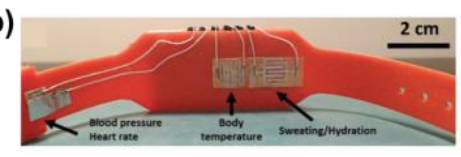

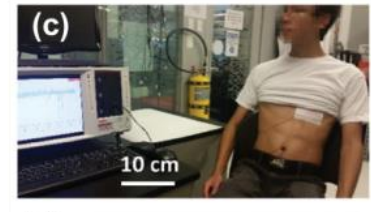
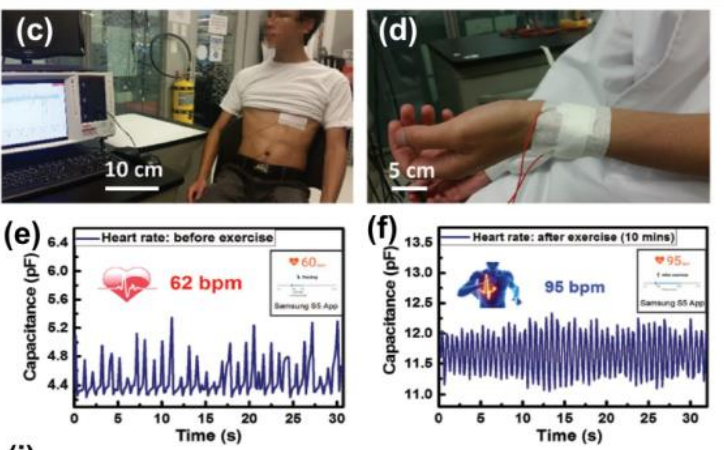

(j)

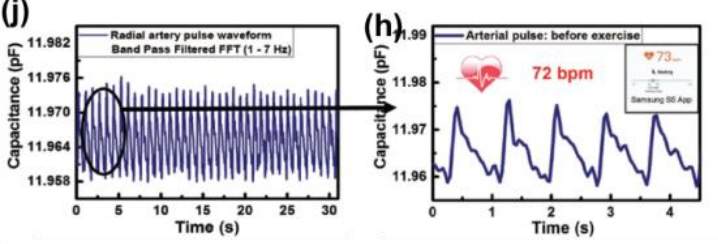

Figure 1.5: Wearable paper-based multisensory platform for simultaneous monitoring. a) Schematic demonstration of a 3D stack of the paper based multisensory health monitoring system. The insets 1 though 5 on the right side show the components of the device which are printed in paper such as RFID, power source printed circuitry processing unit, sensors readout circuitry and multifunctional sensory platform. b) Optical photograph of the sensory platform assembled as a paper watch in contact with the radial artery around the wrist. Figures $c-h)$ show real-time body vitals monitoring experiment including photographs of the setup and the results of characterization of the device in two main locations, on the chest and around the wrist. 


\subsubsection{Wearable Transdermal Drug Delivery Devices}

Transdermal drug delivery offers a more attractive non-invasive administration rout for drugs than the intramuscular and the subcutaneous routes. Skin is the largest organ of the human body; however, the challenge lies on the fact that the outermost layer of the skin, the stratum corneum, acts as a protective layer that limits the diffusion of molecules to the skin. Since the lipid bilayer of the cell membrane has hydrophobic chains on the outside, it is especially difficult for hydrophilic molecules or high molecular weight molecules to pass through this membrane ${ }^{[34]}$. The use of microneedles overcome this challenge by enhancing the delivery of drugs since the microneedles have a length that penetrates the stratum corneum layer and allows for the passage of large and hydrophilic molecules like insulin that normally have low permeability to the skin when applied topically ${ }^{[35]}$. Another advantage of transdermal drug delivery is that is increases the bioavailability of the drug because it offers a shorter route to the bloodstream and it bypasses the gastrointestinal tract and being metabolized the liver. Transdermal delivery routes also result in excellent patient compliance since the method is non-invasive, painless, and easy to administer. The first generation of wearable transdermal drug delivery are diffusion-based in form of drug loaded patches. Since the mechanism relies on passive diffusion, however, their application limited to drugs that are made out of small or hydrophobic molecules. Moreover, the delivery mechanism is considered slow because molecules need time in order to bypass the stratum corneum layer and reach to the inner layer of the skin containing capillary blood vessels. As an example, a 
multifunctional wearable drug-delivery device developed by Kim et al. is shown in figures 1.6a and 1.6b ${ }^{[36]}$. The device contains flexible temperature, strain sensors, electroresistive heaters and resistive random-access memory array. The objective of the device is to monitor muscle activity and provide therapy for people with movement disorders. The strain sensor is able to detect movements patterns by monitoring tension and compression forces produced by the wrist. The release of drug occurs by heating a drug loaded silica nanoparticles membrane. Heating is added to actuate the drug releases. Once the heater is turned on, the physical bonds between the membrane and the drug will weaken allowing for the transdermal drug diffusion to occur ${ }^{[36]}$. Another device that utilizes the use of microneedles was developed by Zhen Gu et.al can be seen in figure 1.6c. The device contains a wearable and stretchable elastomer with the drug inside microgel depots. The actuation mechanism of drug relies on the application of tensile strain by the body. Tensile strain increases the diffusion surface area and triggers drug release due to Poisson's ratio-induced compression on the microgel depots. An array of polymeric microneedles is integrating into the device for insulin delivery ${ }^{[37]}$. Polymeric microneedles, although are highly biocompatible, have limited applications to only drug eluting-patches. Moreover, this mechanism is not fully controlled since the devices will continuously deliver drug due to the continuous movement of the body. Thus, full spatiotemporal control of drug release is not achieved. Another promising wearable device utilizes a graphene doped with gold mesh for sweat glucose monitoring and Metformin delivery. The components of the device include a heater, temperature sensor, humidity sensor, and tridecanoic acid coated polyvinylpyrrolidone microneedle shown in 
figures 1.6e and 1.6f. Upon detecting the glucose level in the sweat, the drug delivery can occur by heating thermally melting the tridecanoic acid coated in the microneedles ${ }^{[38]}$. The limitation of such devices is that the microneedles are bioresorbable and thus they are not appropriate for long term delivery.

(a)

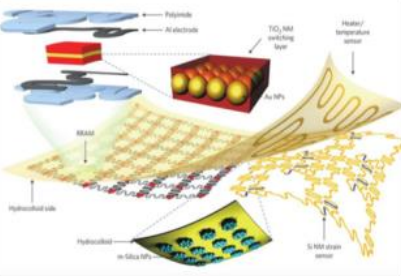

(b)

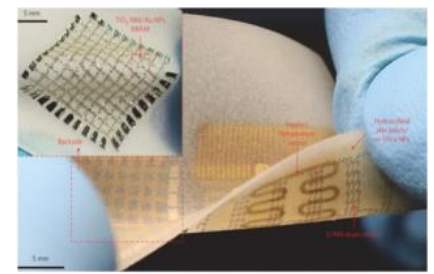

(c)

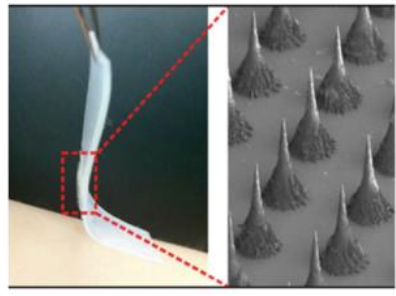

(d)

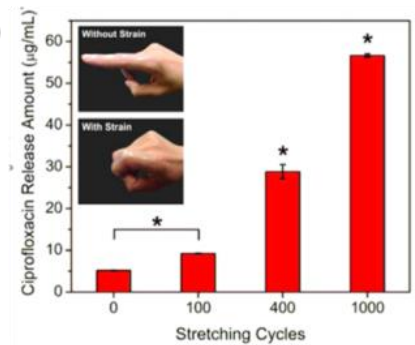

(e)

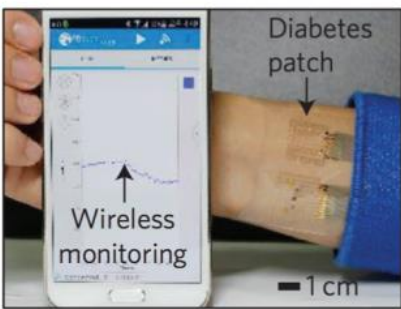

(f)

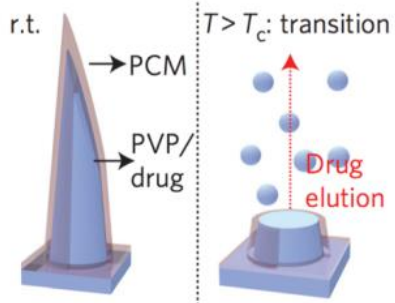

Figure 1.6: Wearable transdermal drug delivery systems. a) and b) a thin wearable device for continuous monitoring of muscle activity and movement disorder therapy) a stretch-based actuated drug delivery device featuring drug microgel depots and microneedles integrated into elastomer gel for stretch- controlled drug released) Passive drug release during different number of finger flexion cycles. e) A glucose monitoring and drug-delivery device showing real-time wireless capabilities. f) Schematic demonstration of bioresorbable drug-loaded microneedles.

\subsection{Microfluidics}

Microfluidics is the science and technology of fluid manipulation at the microscale in a complex network of microchannels, valves, reservoirs, and pumps. It is a recently emerging field that combines engineering, physics, chemistry, biology, and nanotechnology ${ }^{[39]}$. The behavior of fluid at the microscale is very different from the macro scale because of the changes in scaling laws. These phenomena include capillary forces, surface tension, laminar flow, and others. Microfluidics take advantage and leverage these phenomena that emerge at this scale to enable high throughput 
processes ${ }^{[40]}$. Historically, microfluidics borrowed the fabrication techniques and process from the well-developed silicon microelectronics, and the microelectromechanical systems (MEMS) technologies. In terms of materials, however, silicon is not the most common material used for microfluidic devices. Polydimethylsiloxane (PDMS) is the most commonly used material for the fabrication of microfluidic devices. PDMS has many attractive properties that make it excellent for microfluidics applications. It is transparent, which make it easier to visualize the flow of colored fluid inside a complex network of channels. It is easier and faster to fabricate than silicon. Moreover, it is cheaper and more robust than silicon ${ }^{\left[{ }^{39]}\right.}$. Moreover, microfluidics offers several remarkable advantages as it deals with fluids of small volumes. It provides rapid analysis, high sensitivity, high scalability, portability, small footprint, reduced power consumption, reduced cost, and reduced waste. Applications are plenty, for instance, point of care diagnostics, drug delivery, analytical devices, industrial applications, environmental applications, and many others ${ }^{[41]}$.

\subsubsection{Mixing in Microfluidic Systems}

Micro-mixing within microfluidic devices is a crucial process that is used for many applications ${ }^{[42]}$. It is used in Lab on chip (LOC) diagnostic and micro total analysis systems $(\mu \mathrm{TAS})$ that to enable high throughput analysis of fluids. Micro-mixing can be categorized into two main types, passive, and active mixing. Passive mixers depend purely on the pumping energy and diffusion of particles while active mixers use additional

pumping forces such as pressure, electrowetting, ultrasound and others. ${ }^{[42]}$. Examples of 
passive micro mixing geometry are $\mathrm{T}$ micromixers, $\mathrm{Y}$ micromixers, chaotic advection micromixers and droplet micromixers ${ }^{[42]}$. Mixing in microfluidics environments leverage the advantage of the unique properties of the fluid at the micro scales. It provides a highly controlled process and controlled product composition in a rapid process. It also enables the production of nanoparticles, microparticles and colloidal systems. Mixing within microfluidics also provide spatial and temporal control of different process and reactions by carrying out the reaction in a multi-step precise fashion. The advantages of mixing in microfluidics devices are extended to drug delivery applications since mixing is capable of the generation of micro-carriers that can be used as drug carriers. Microcarriers are used to improve the efficacy, selectivity, bioavailability, and safety of conventional oral and intra-venous administered drugs ${ }^{[43]}[44][42]$. Microfluidics drug delivery devices offer advantageous properties over conventional drug administration methods. The unique fluid flow properties that emerge at the microscale can be leveraged to prepare and deliver more effective forms of drugs and therapeutics to the human body. It can also be integrated with non-invasive painless administration methods through an array of skin microneedles that are seamlessly integrated into these drug delivery systems.

\subsection{Closed-loop Transdermal Drug Delivery Systems}

Most of the existing drug delivery platforms focus on single drug delivery of one type of medication or drug. In addition, the delivery is an active task that has to be triggered at a specific time with a specific predetermined dose ${ }^{[45]}$. A drug delivery device, however, with

the integration of skin sensors can provide automatic on-demand spatiotemporal control 
over drug preparation and delivery. Together, wearable devices that include sensors for continuous physiological signals as well as drug delivery components represent the next generation of customizable feedback personalized therapy where the patient is taking out of the loop to achieve a closed loop smart personalized therapy. Realization of precision medicine necessitates the need of biosensors and actuators to enable the spatiotemporal control of the administered dose of drug as a response to real-time physiological changes of the patient to evaluate the condition of the disease and the efficacy of therapy being delivered. Advancement in soft and stretchable bioelectronics enabled the realization of conformal wearable bioelectronics for drug delivery with incredible functionalities. Sensing elements provide information about the electrophysiological and biochemical signals of the body, actuating elements provide energy to deliver drug into the body on demand. In a further step, the sensors would collect information that assess the efficacy of the delivered drugs. The simultaneous integration of both wearable biosensors and drug delivery devices offer a novel and a closed loop feedback personalized medicinal platform. We report a flexible microfluidics drug delivery device for on-demand personalized therapy. The device includes a flexible multi-reservoir microfluidics layer, flexible and conformal heating elements, skin sensors, processing and power units. The platform is able to mix different type of drugs with varying viscosities, which with the appropriate fluid flow parameters, can be used to formulate drug microcarriers that improve the efficacy, bioavailability and uptake of the drug in the body. Applications are not only limited to the delivery of daily multi-vitamins and minerals; the platform can also be used as more compliant alternative to conventional wearable drug delivery systems 
such as bulky invasive insulin pumps and wearable injectors. The conventional delivery methods include hypodermic needles, pill and transdermal drug delivery methods like diffusion-based patches. The limitations of these methods include invasiveness, poor control and they can be ineffective. The goal is to improve and control drug release, increase effectiveness, decrease side effects. For the drug release, there are different

drug release mechanisms of drug delivery devices. Some devices are controlled by diffusion, and some are solvent controlled, and others are based on erodible components. Regulated devices are devises with externally applied trigger in order to turn on or turn of the release. These devices can be electrically, mechanically or magnetically triggered. The active reservoir devices that are triggered with an actuator to turn on the drug release although can be complex to fabricate in some cases and require power supply, they provide much more control of drug release over conventional methods of drug delivery. The challenge still exists for the development of a an autonomous closed-loop dynamic wearable drug delivery system that is responsive to accurate physiological conditions for long period of times. Such robust system must meet a number of requirements that are discussed in the next section.

\subsubsection{Requirements for Transdermal Drug Delivery systems}

With the recent advancements in wearable biosensors and actuators, there is a need for a wearable personalized drug delivery platform that provides controlled and feedbackbased transdermal drug release. The device can be integrated with already developed wearable sensors, such as the one that have been previously presented by our group ${ }^{[4][33]}$, 
to continuously monitors and collects physiological signals of the body and produce actionable information in the form of therapy. Such personalized devices are considered the next generation of transdermal drug delivery devices. The requirements for such wearable system include the following: i) spatiotemporal control and precise drug release, ii) simultaneous delivery of multiple drugs, iii)flexible architecture, conformity and conformability, iv) affordable fabrication techniques with capability of mass production at low cost, v) Low power consumption, vi) non- invasive transdermal delivery, viii) improved drug efficacy, ix) personalized and tailored for each individual's needs, $\mathrm{x}$ ) decreased side effects.

\subsection{Research Objectives and Outline}

\section{Objectives}

The primary objective of this project is to develop a transdermal drug delivery platform that is, wearable, personalized, automated, highly conformal to the skin, cost-effective, simple to use, power efficient and effective. A secondary goal was to optimize the performance of the system by addressing critical parameters that effect the accuracy of the controlled drug release ad its power consumption. 


\section{Thesis Outline}

The chapters included in this thesis are organized as follows:

In chapter 1, an overview of the state-of-the-art wearable devices are discussed including sensors and drug delivery devises. A brief background about the field of microfluidics, its capabilities and applications in the context of drug delivery and health monitoring are introduced. In chapter 2, the design parameters of the personalized drug delivery platform are discussed. The materials and fabrication process of the system are described and discussed. The fabrication techniques in this chapter include laser patterning, soft lithography, PDMS casting, 3D printing, sputtering, electroplating and oxygen plasma etching. Design layouts of the separate layers of the device, recipes and tools used will be included in this chapter.

In chapter 3 , the characterization and optimization of the different components of the platform are presented including the heater, actuators, microfluidics reservoir layer and wearable skin sensors. This chapter also includes the results of the system integration with microneedles and closed loop feedback experiment.

Chapter 4 discusses the major concluding remarks and potential future work of this project. 


\section{Chapter 2 Device Fabrication}

The wearable platform is composed of three main layers - the microheater layer, the expandable composite layer, and the microfluidics reservoirs layer. Overall, simple and rapid fabrication techniques were adopted to enable efficient mass fabrication of the device and expand its applications of in low-resource environments. In the following sections of this chapter, details about the fabrication process of each layer will be discussed.

\subsection{Heater Fabrication}

The actuation layer that is used as a pumping mechanism for the microfluidic device is composed of a thermoresponsive polymer that expands upon heating. Details about the expandable layer will be discussed in the next section, but this section is about the fabrication of the heating element that is used to actuate the polymer. The heaters are

located underneath the expandable polymer layer. Together, they form the actuation unit of the device where the microheaters will allow for selective expansion of some critical areas of the expandable composite to expand. The liquid reservoirs layer resides above the crucial areas of the expandable composite, which will enable the instance displacement of the liquid from the reservoirs upon turning on the heaters.

\subsubsection{Microheater Array}


A set of microheaters need to be designed and fabricated in order to provide the actuation mode for the expandable polymer Expancel ${ }^{\circledR}$. The critical temperature in which Expance ${ }^{\circledR}$ experiences a volumetric expansion is around $76-81{ }^{\circ} \mathrm{C}^{[46][47]}$. Thus, the heater must provide the required amount of thermal energy to cause expansion and at the same time consume the least amount of power as possible.

\subsubsection{Operating Principle of the heaters}

The fundamental operating principle of the heater is based on joule heating or resistive heating in which the passage of electric current into a conducting element produces heat ${ }^{[48]}$.The conducting element is a thin metal electrode fabricated on a substrate with high resistivity. Upon heating, a good stable substrate should disburse heat over the area of the heater. The heating power of the heater can be calculated when voltage is applied in one side of the contact of the heating element (resistor), and the other side of the contact of the heating element is grounded. The heating power equation is as follows [49]

$$
\begin{array}{r}
P=I^{2} R \\
\text { or } \\
P=\frac{V^{2}}{R}
\end{array}
$$

Where I is the applied current, $\mathrm{V}$ is the applied voltage and $\mathrm{R}$ is resistance of the heater which is calculated as follows

$$
R=\frac{\rho L}{A}
$$

where $\rho$ is the resistivity of the material, $L$ is the length of the track of the heater and $A$ is 
the cross sectional area of the heating element material.

The heating power equation can be re-written as

$$
P=\frac{V^{2} A}{\rho L}
$$

Thus, by designing the length of the heating element and the thickness which corresponds to the cross-sectional area, the power consumption can be determined.

\subsubsection{Design}

The objective is to design and fabricate a microheater that will provide the required critical temperature and good temperature distribution over the area of the heater while consuming the least amount of power. To achieve this, it is imperative to find a design that will minimize heat conduction losses by having good and efficient thermal isolation. In order to find the optimal heater design that will provide the mentioned features, different heater geometries were investigated. Previous work in the literature has been performed by other groups to try to find the optimal heater designs for different applications such as gas sensors and other applications ${ }^{[50]}$. Some of the most commonly used design patterns are simple meander pattern, spiral pattern and fan-shaped pattern. These patterns were chosen to be fabricated and tested to determine which design provide the optimal performance for the need for this project. For comparison purposes, the area covered by the heater was fixed to be the same across all three patterns $7 \times 7$ $\mathrm{mm}^{2}$ and the cross-sectional area A was fixed to be the same by using the same thickness of heating material $8 \mu \mathrm{m}$ and tge same width of the electrode of $520 \mu \mathrm{m}$. The pattern if each design with dimensions are shown in figure 2.1. Additional circular-spiral design that 
was also fabricated and tested is seen in figure 2.2.
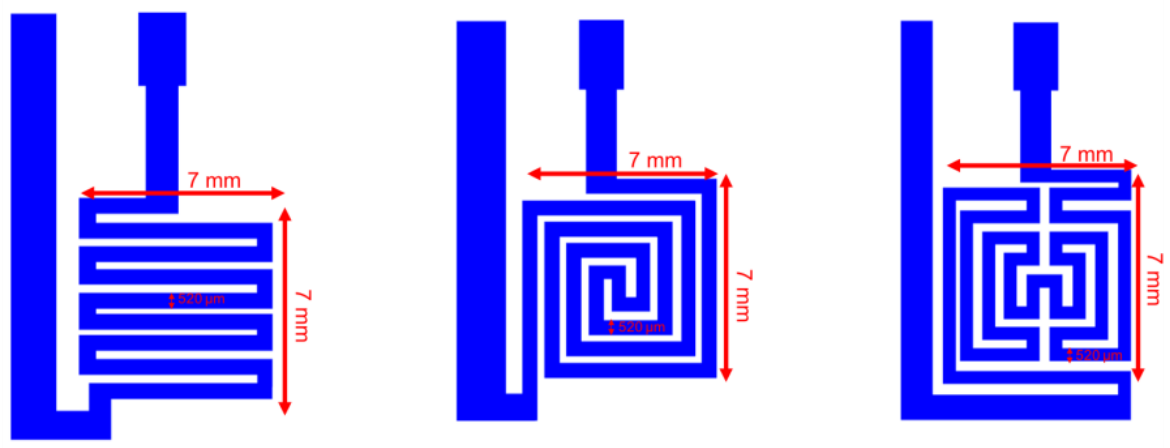

Figure 2.1: Microheater Patterns. Designs of the different microheaters geometries. Meander, spiral and fan-shaped heater

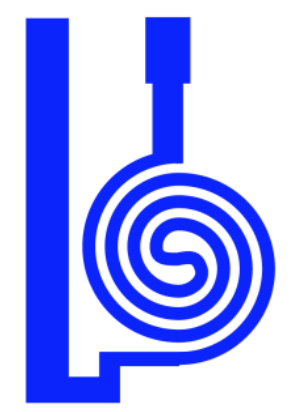

Figure 2.2: Circular-spiral design

\subsubsection{Materials}

The advantage of miniaturized microheaters with a micrometer thick substrate minimizes the amount of lateral heat lost that might occur into the substrate and as a result decreasing the power consumption. In this case, however, it was also important for the substrate to be thick enough to withstand the mechanical force that will be exerted when the expandable polymer expands. Thus, a thickness of $25 \mu \mathrm{m}$ was chosen as the thickness of the polyimide substrate. It is strong enough not to rupture or fold while the polymer coated above is expanding, and it is still flexible and bendable for it to conform into the 
skin. The substrate material chosen was a polyimide (PI) film. It was chosen for its desirable properties as a substrate such as thermal stability, long-term stability and low

manufacturing cost ${ }^{[51]}$. The metal that was chosen to construct the thin heating element is copper for its excellent conductivity and flexibility among other commonly used metal and also its cost-effectiveness. Since the size and area of the heater affect the power dissipation, it was imperative to use the same size and area for the al the heater in order to compare their performance more accurately.

\subsubsection{Fabrication Process}

The heater layer was fabricated by electroplating $8 \mu \mathrm{m}$ of copper on a $25 \mu \mathrm{m}$ thick polyimide film (Good Fellows) sheet. Electroplating was chosen in this case as it is more efficient in terms of time than sputtering in deposition layers thicker than $1 \mu \mathrm{m}$. The time needed to electroplate a 4-inch wafer sized PI sheet with $8 \mu \mathrm{m}$ of copper was 45 minutes using a current of .210 A. Prior to electroplating, the surface of the PI substrate was treated with oxygen plasma to activate the surface and improve adhesion. The oxygen plasma treatment parameters ware a chamber pressure of $20 \mathrm{~Pa}$, a temperature of $20^{\circ} \mathrm{C}$, and a flow of $30 \mathrm{sccm}$ of oxygen for 3 minutes. After that, a thin $150 \mathrm{~nm}$ gold layer was deposited using sputtering to act as a seed adhesion layer for electroplating. After that, the heater design pattern was transferred by laser ablation method using $1.06 \mu \mathrm{m}$ ytterbium-doped fiber laser (PLS6MW Multi-Wavelength Laser Platform, Universal Laser Systems). The laser settings that were optimized for the pattering recipe are $30 \%$ power, 
$40 \%$ speed of the total capacity of the laser. Schematic of the discussed fabrication process is shown in figure 2.3.

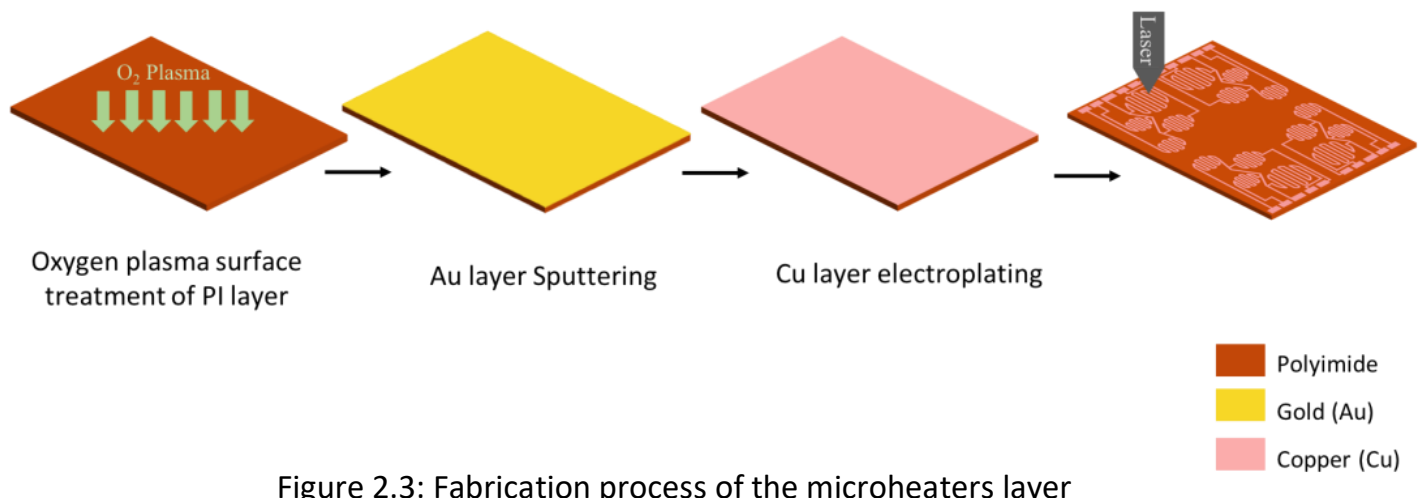

\subsection{Actuator Unit Fabrication}

\subsubsection{Expancel Microspheres}

The concept of the platform is that it contains reservoirs of well-defined capacities. This means that the delivery of the drug is restricted to a discreet amount of liquid volume defined by the volume of each reservoir. This concept enables dosage control and active, precise delivery. This also necessitates the need for an actuating unit that can sufficiently displace the liquid out of the reservoir acting as the pumping unit of the device. Because the layer that contains the drug component of the platform was designed to be disposable to decrease overall complexity, it was appropriate to choose an actuator that is irreversible and enables enough volumetric expansion to displace and infuse liquid from the reservoir. Expancel microspheres were chosen as the active actuating material for the platform for its many desired features. Expancel microspheres are small polymeric 
particles that contain thermoplastic shell encapsulating isobutane hydrocarbon gas. When heated up to a certain critical temperature of $\sim 80$ ac, the pressure inside the particle increases from the gas and the shell starts to soften allowing for a dramatic volumetric expansion of the microspheres. Figure 2.4 below shows a schematic of the principle of expansion of expancel microspheres from AkzoNobel (Amsterdam, The Netherlands) ${ }^{[46]}$.

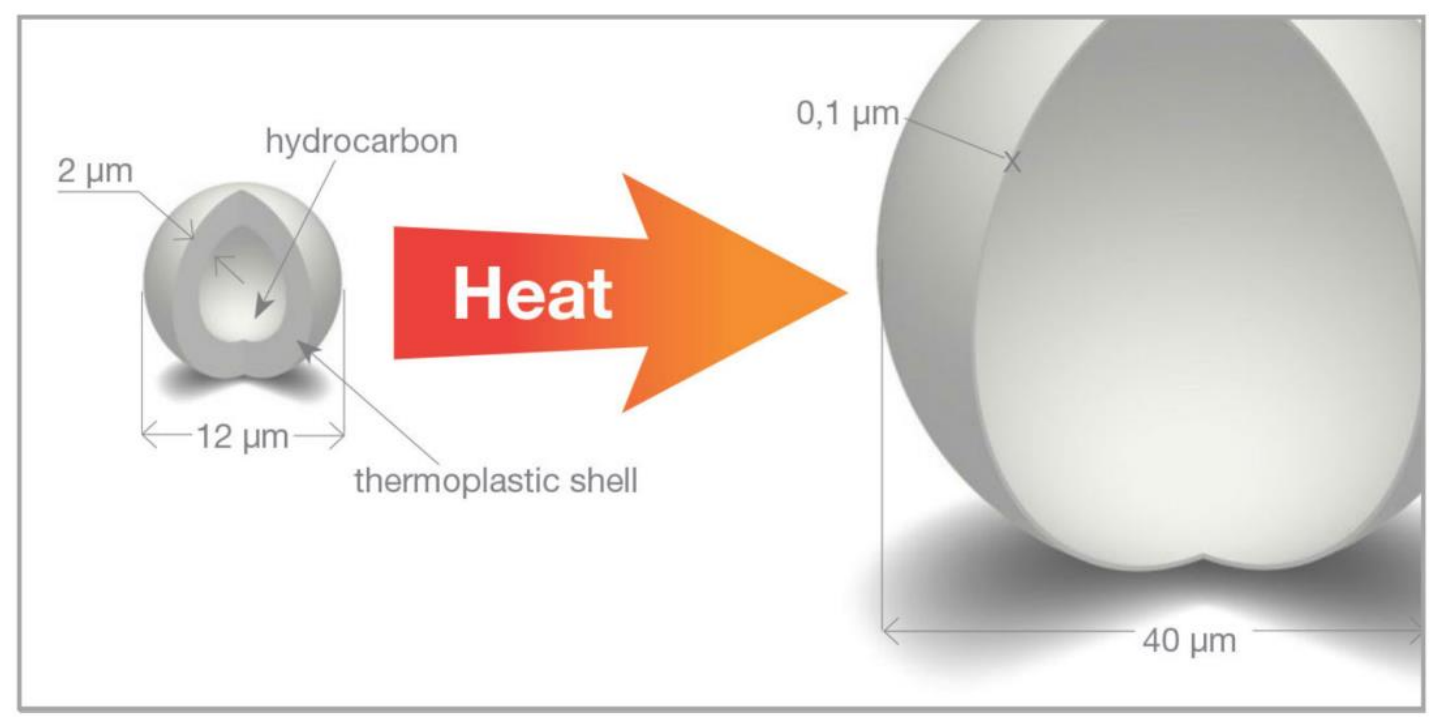

Figure 2.4: Schematic illustration of the physical principle behind the volumetric expansion of Expancel ${ }^{\circledR}$ microspheres

In general, phase-transition based actuators are ideal in this case since they provide the highest liquid displacement ${ }^{[52]}$. Moreover, expancel microspheres are irreversible in their action which is appropriate for a single-use purpose device that decreases the overall decreases complexity of the system. Thus, expancel was determined to be an appropriate liquid displacement mechanism in this application. 


\subsubsection{Expancel-PDMS composite}

Exapncel microspheres have been used previously as an actuating element for microfluidics devices for various applications such as single-use pumps, microinjectors, and blood coagulation testing. A common method of using expancel microsphere is by embedding the microspheres into silicon elastomer called Polydimethylsiloxane (PDMS) made of Sylgard ${ }^{\circledR} 184$ (Dow Corning Corp., Midland, MI, USA) ${ }^{[53]}$. Mixing expancel microspheres with PDMS forms a viscous composite that can be easily deposited, or spin coated over a heater or other surfaces to form a thin uniform layer.

\subsubsection{Operating Principle of the expancel as an actuator}

The microheaters will be placed underneath the expancel composite layer which is located underneath the fluidics reservoirs. When the heater is turned on, heat will be generated and expancel composite layer consequently will experience irreversible volumetric expansion which will displace the liquid and release it through the microfluidics channel. Figure 2.5 presents a schematic showing the operation principle of the device utilizing the expansion of expancel composite to displace the fluid from the reservoir to the outlet through microchannel and microneedle. Figure $2.5 \mathrm{a}$ shows the state of the device before turning the heater on. After triggering the heater, the expendable composite will expand and push the fluid out as see in figure $2.5 \mathrm{~b}$. Figure $2.5 \mathrm{c}$

demonstrates the application of the device on the human skin and the fluid infusion to the skin layer through a hollow microneedle. 


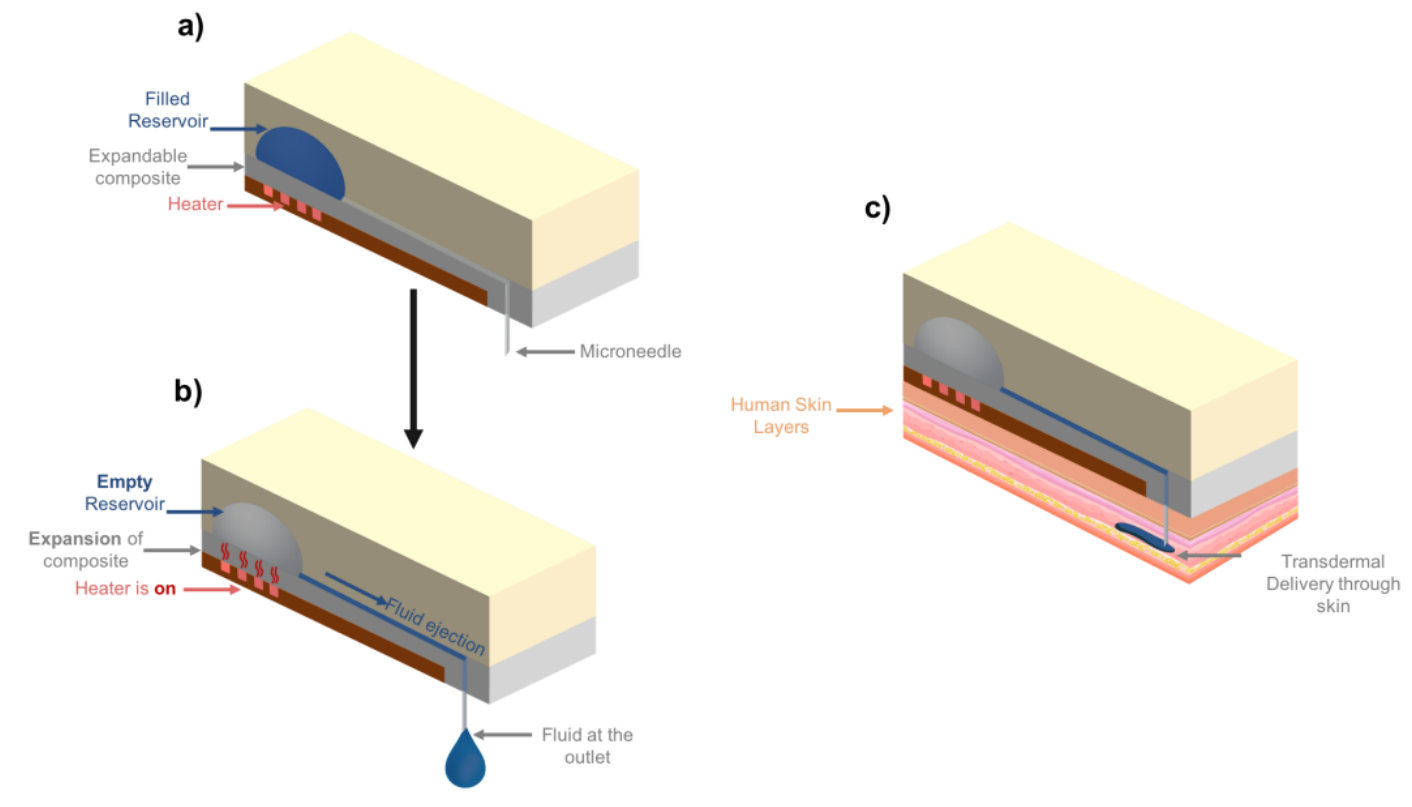

Figure 2.5: Schematic illustration of the operation principle behind of the device. a) intital state of the device. $b$ ) the state of the device after turning on the heater and expansion of the composite pushing liquid to the outlet. c) Demonstration of the application of the device on human skin.

\subsubsection{Fabrication Process}

The expandable expancel polymer microspheres powder Expancel 031 DU 40 was purchased from AkzoNobel in its powder form. The expandable layer was prepared by mixing expancel powder with polydimethylsiloxane (PDMS) (Sylgard 184 silicone elastomer kit) using a 2:1 ratio of PDMS to expancel in a vacuum mixer. After that, the prepared mixture was then spin coated on top of the heater layer with a spin coating speed of 600 round per mint for 60 seconds. In order to obtain the thin layer of the expandable PDMS-expancel composite, the layer was cured in an oven at $70{ }^{\circ} \mathrm{C}$ for 2 hours. The thin layer has a thickness of around $280 \mu \mathrm{m}$. 


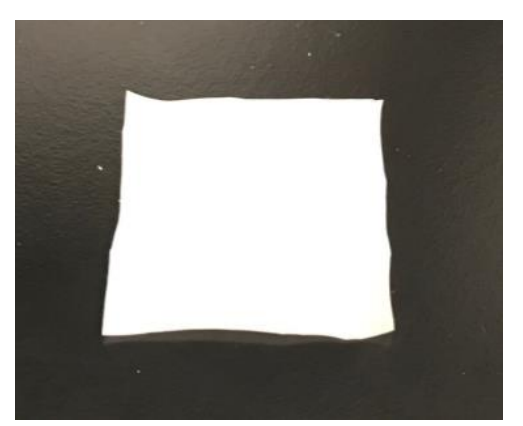

Figure 2.6: Optical photograph of a fabricated expandable composite layer after releasing it from the wafer.

\subsection{Microfluidics Layer Fabrication}

The goal is to develop a platform that is able to provide both drug formulation and delivery. Thus, the microfluidics layer was carefully designed to meet these two aims. For the delivery part, microchannel of width and depth of 150 lm and 100 am respectively was designed to carry out the displaced liquid drug from the reservoir to the outlet of the device. The outlet is connected to a microneedle for complete transdermal delivery. For drug formulation, the microfluidics device is featuring a micro-mixer that is capable of mixing different fluids. Mixing is one of the significant properties that is crucial for microfluidic devices for drug formulation and synthesis purposes. The geometry of the micro-mixer was chosen and optimized to ensure efficient passive mixing of fluids. In the following sections, more details about the geometry of the mixer, the microchannels, and the reservoirs are discussed.

\subsubsection{Design \& Layout}

There are two main designs of microfludic reservoirs layers that were fabricated and used for this device. The first design features a total of 6 hemi-spherical reservoirs with the 
following capacities $261.8,134.04$, and $56.55 \mu \mathrm{L}$. The reservoirs are connected via microfluidics channels with a cross section of $150 \times 150 \mu \mathrm{m} 2$ (width $\mathrm{X}$ depth). The channels also contain individual micro-mixers as well as a final mixro-mixer that is connected to the outlet. The purpose of the small micro-mixers is to prevent back flow from other reservoirs while the purpose of the major micro-mixer is to facility mixing of different liquids. The design layout Is demonstrated in figure 2.7.

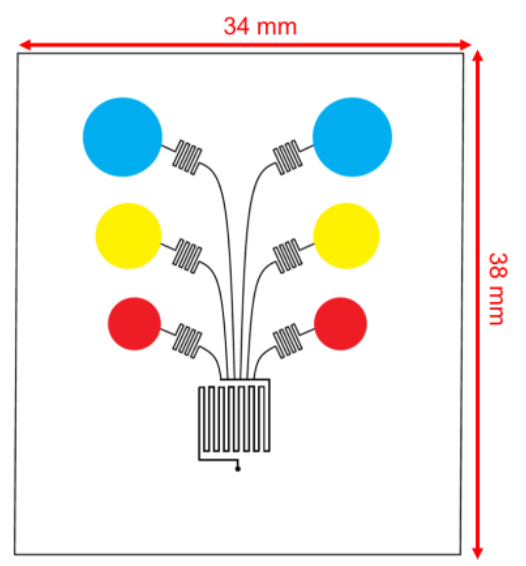

Figure 2.7: Schematic of design 1 of the microfluidics reservoir layer.

The second design is more complex as it contains 16 revivors with 4 different capacities of $452.4,261.8,134.04$ and $56.55 \mu \mathrm{L}$. The layout of the second design is seen in figure 2.8.

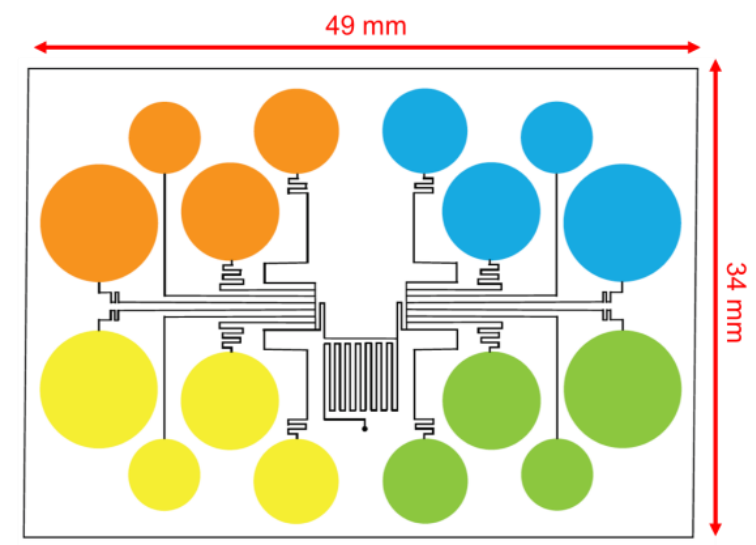

Figure 2.8: Schematic of design 2 of the microfluidics reservoir layer. 


\subsubsection{Micromixer Geometry}

One objective of the platform is to have mixing capabilities. Thus, it was important to understand the mechanisms that governs mixing at the micro level. It is also important to understand the nature and the behavior of fluid flow inside microfluidics devices. The nature of fluid flow in microfluidics is non-turbulent laminar flow. To understand it further, the concept of Reynolds Number will be discussed. Reynolds number is a dimensionless number that represents the relationship between inertial forces and viscous forces ${ }^{[54]}$.

$$
R e=\frac{\text { Inertial forces }}{\text { Viscous forces }}=\frac{\rho v^{2} L^{2}}{\eta v L}=\frac{\rho v L}{\eta}
$$

where $\rho$ is the density of fluid, $v$ is its velocity, $\eta$ is its viscosity and $L$ is the characteristic length of the channel. There are three cases for Re number which determine whether the flow is laminar, turbulent or in an intermediate state in between.

$0<\operatorname{Re}<1$ Laminar flow, viscous forces dominate

$1<\operatorname{Re}<2300$, intermediate flow

$2300<\operatorname{Re}<\infty$ Turbulent flow, inertial forces dominate

In the microfluidics world, the flow is always laminar due to the extremely small characteristic length $(L)$ of the channel. When two different fluids are in contact with each other, thermally induced random Brownian movements of their molecules will induce passive mixing. Since fluid flow in microfluidics devices is laminar, passive mixing is highly 
depended on molecular diffusion and advection ${ }^{[42]}$. Diffusion of molecules occur as a result of the Brownian motion across the interfacial contacts between the fluids. Fick's law of diffusion is defined as

$$
j=-D \frac{d_{\varphi}}{d x}
$$

where $j$ is the diffusion flux, $\mathrm{D}$ is the diffusion constant which is characteristic for a given particle, $\varphi$ is the concentration of particles and $\mathrm{x}$ is the position of the particle ${ }^{[42]}$. For spherical particles, D can be defined as

$$
D=\frac{k T}{6 \pi \mu R}
$$

Where $\mathrm{k}$ is Boltzmann constant, $\mathrm{T}$ is the temperature, $\mu$ is the viscosity of fluid and $\mathrm{R}$ is the radius of the particle. Diffusion has nonlinear relationship between the diffusion time and the distance particle travels, it is a quadratic relationship ${ }^{[42][55]}$. A simple model of the relationship can be defined as

$$
x=\sqrt{2 D t}
$$

where $\mathrm{x}$ is the average distance a diffusing particle covers within time $t$. I this simple equation, temperature is being taking care of in the diffusion constant term. In microfluidics systems, $x$ is represented as the width of the liquid fluid that is going to be mixed along the channel. Thus, the time required to diffuse through half the channel width (W) can be calculated as

$$
t=\frac{x^{2}}{8 D}=\frac{W^{2}}{8 D}
$$

In this experiment, food dye is being used to carry out the mixing experiment and proof 
the concept. The diffusion coefficient of food coloring in water at room temperature is $2 \times 10^{-6} \mathrm{~cm}^{2} / \mathrm{s}$ and the width of the channel is $150 \mu \mathrm{m}$. Thus, the time needed for a particle to diffuse through half the channel width $\mathrm{W}$ is calculated to be

$$
t=\frac{W^{2}}{8 D} \frac{(.015 \mathrm{~cm})^{2}}{8\left(2 \times 10^{-6} \mathrm{~cm}^{2} / \mathrm{s}\right)}=14.0625 \mathrm{~s}
$$

Knowing the time needed for a complete mixing, the required length of the channel can be calculated. The volumetric flow rate used for the platform is $5 \mu \mathrm{l} / \mathrm{min}$ or $8.33 \times 10^{-5} \mathrm{~cm}^{3} / \mathrm{s}$. Thus, the velocity can be calculated from

$$
v=\frac{Q}{A}
$$

Where $Q$ is the volumetric flow rate, $A$ is the cross-sectional area. The cross-sectional area of the channel is $.015 \mathrm{~cm} \times .015 \mathrm{~cm}=.000225 \mathrm{~cm}^{2}$

Thus

$$
v=\frac{Q}{A}=\frac{8.33 \times 10^{-5} \mathrm{~cm}^{3} / \mathrm{s}}{.000225 \mathrm{~cm}^{2}}=.37 \mathrm{~cm} / \mathrm{s}
$$

Length of the channel needed is $=14.0625 \mathrm{~s} \times .37 \frac{\mathrm{cm}}{\mathrm{s}}=5.203124 \mathrm{~cm}=$

\section{$52.03125 \mathrm{~mm}$}

This is the main design constraint needed to bed applied on the design of the micro mixer, which is that its total length needs to be $52.03125 \mathrm{~mm}$ or more. This design constrain will ensure sufficient mixing in the structure. To achieve this, a meandering design of microchannel is an excellent candidate to be used. Meander design provide added length to the channel which offers enough length for the interfacial contact between the two fluid streams to achieve efficient diffusion and induce chaotic advection. The proposed 
design can be seen in figure 2.9a below. The total length of mixromixer is $85 \mathrm{~mm}$ which is more than the needed length of $52 \mathrm{~mm}$ to ensure complete mixing under different conditions.

a)

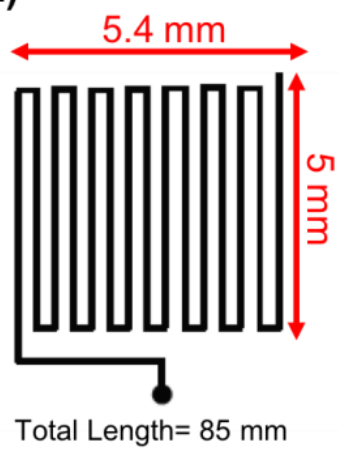

b)

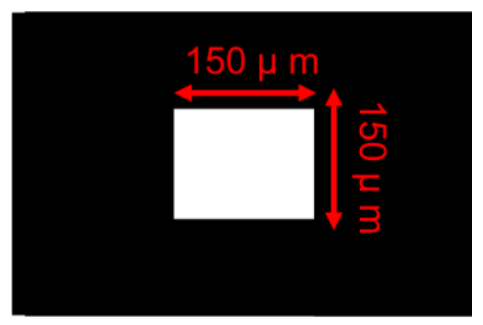

Figure 2.9: Micromixer design. a) Meander pattern used for the micromixer with its dimensions.

b) cross section schematic of the microfluidic channel showing its width and depth.

\subsubsection{Reservoir Geometry}

The reservoir geometry of most of the reservoir-based drug delivery systems is a cube or cylinder. Both of these structures have planar surfaces which can be explained by the limitation of the two-dimensional microfabrication processes. The shape of the reservoir of the drug delivery platform affects the performance of the device, specifically the expansion profile of expancel and the amount of fluid being depleted out of the reservoir. In order to investigate this effect, four different geometries that construct the shape of the reservoirs were chosen which are a cylinder, hemisphere, truncated-cone, and square. The structural designs of the four geometries can be seen in figure 2.10 as well as optical photographs of the fabricated reservoirs. 
a)

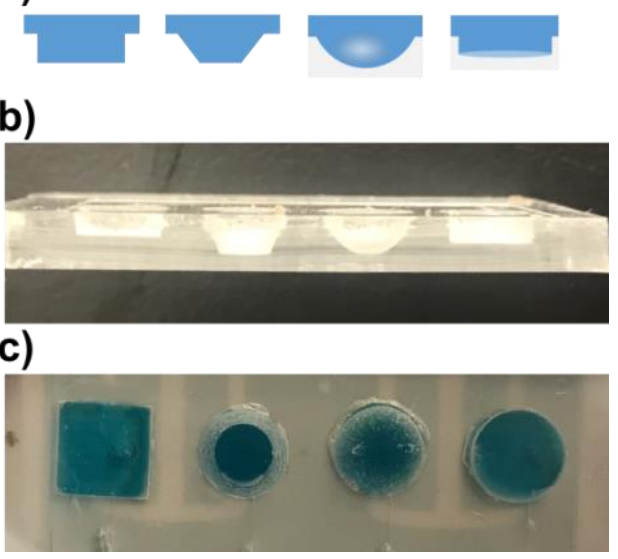

Figure 2.10: Reservoirs Geometry. a) Schematic illustration of the designs of the four different reservoirs shapes to be tested. b) Optical image of the fabricated reservoirs (side view). c) Optical images of the reservoirs after filling them with fluid (top view)

\subsubsection{Materials}

PDMS is the most widely used material for microfluidics devices. It was chosen for its attractive properties such as mechanical flexibility, stretchablitiy, robustness, low cost, and rapid fabrication techniques [32].It is biocompatible and does not cause any irritation to the skin. It is also fabricated using easy and rapid techniques such as molding and casting.

\subsubsection{Fabrication Process}

The microfluidic reservoirs layer was fabricated using PDMS casting on a 3D printed master mold. The microfluidics layer is fabricated by 3D printing a master mold of the microfluidics layer. The printer that was used is ProJet MJP 2500 plus high-quality printer (3D systems, USA). The microfluidics chip structures of channels and reservoirs were designed using computer-aided design (CAD) software SolidWorks (Dassault Systèmes SE,

France). The 3D CAD data were converted and sliced into layers and saved as STL format, a format that is compatible and widely used in most commercial 3D printers. After 
printing, the 3D master mold was cleaned using the Ease clean system of the printer to remove the structural wax supporting layers. Additional cleaning was performed by washing the 3D printed mold with ethanol to remove any wax residues. After cleaning, the 3D printed mold was treated with silicone ease-release spray to facilitate the demolding process later. Figure 2.12 shows the 3D printed mold after cleaning and drying. A PDMS mixture was prepared and casted on the master mold and inserted into a vacuum chamber for 20 minutes to remove any existing bubbles in the polymer. Later the PDMS was cured in the oven at $70{ }^{\circ} \mathrm{C}$ for 2 hours. It was removed carefully using razor blades on the edges to prevent the rupture of the layer during the demolding process. The PDMS microfluidics layer was coated with $3 \mu \mathrm{m}$ layer of parylene $\mathrm{C}$ to prevent fluid evaporation for long term storage. The fabrication process flow is illustrated in figure 2.11.An alternative fabrication method of microfluidics layer was also performed. PDMS was casted on a 1-mm dishes and cured in the oven at $70{ }^{\circ} \mathrm{C}$ for 2 hours. After curing, the PDMS was directly patterned via CO2 PLS6.75 laser universal systems and using hairline setting mode with extremely low laser powers. After ablation, the layer device was brushed with soapy water, rinsed with isopropanol, inserted into a sonicator bath for 2 minutes and finally blow dried with nitrogen air to guarantee that no residue left from the ablation process which might block the channels. 


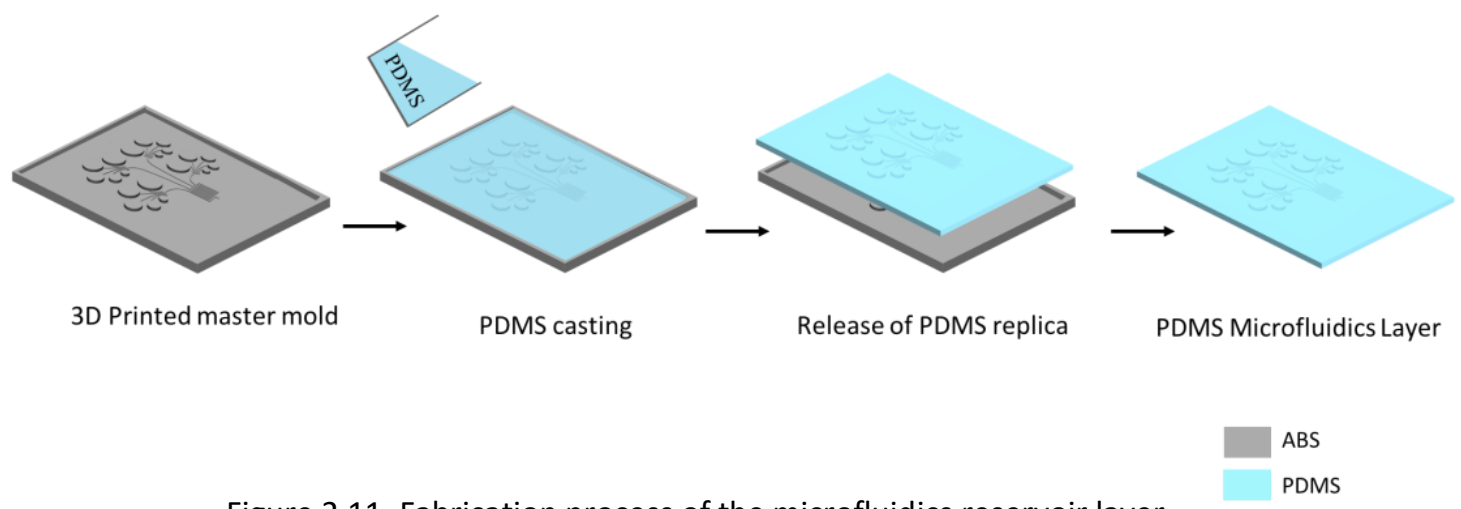

Figure 2.11. Fabrication process of the microfluidics reservoir layer

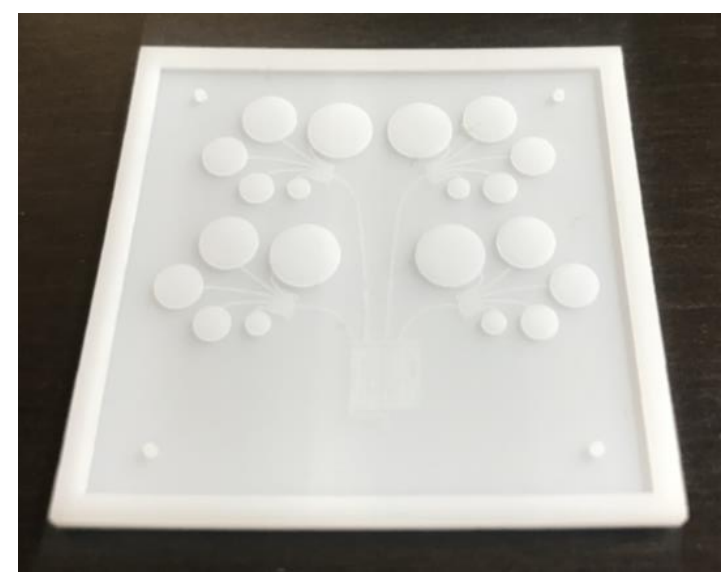

Figure 2.12: Optical photograph of the 3D printed master mold.

\subsection{Wearable Sensors Fabrication}

\subsubsection{Operating principle of Temperature and Humidity Sensor}

In order to prove the concept of the closed-loop feedback system, it was imperative that a couple of wearable sensors are fabricated and integrated into the system. Two types of sensors that have already been developed previously by our group were chosen which are temperature and humidity sensors ${ }^{[56][51]}$. The fabricated temperature sensor is a resistive-based sensor where the change in electrical resistance of a conductive material corresponds to change in the temperature. This change in resistance upon varying the 
temperature will occur because the phonon in the metal lattice structure will thermally vibrate upon heating, which will increase the distance between each atom in the metal leading to weaker conductance of electricity in the metal. This decrease in conductivity translates into an increase in electrical resistance ${ }^{[57]}$. The relative change in resistance as a function of temperature is commonly represented by the value of the temperature coefficient of resistance (TCR) which is defined as

$$
T C R=\left(\frac{\Delta R}{\Delta T}\right) / R
$$

where $\Delta R$ is the change in resistance, $\Delta T$ is the change in temperature, and $\mathrm{R}$ is the initial resistance of the sensor.

The fabricated humidity sensor is a capacitive-based sensor where the change in electrical capacitance of a conductive material corresponds to change in the humidity level. The copper metal sensing element is patterned on a polyimide substrate. The polyimide substrate acts as the dielectric material separating two paralleled metal electrodes/plates. As water molecules adsorb to the surface of $\mathrm{PI}$, its permittivity increases. Thus, if the moisture level in the air rises, PI permittivity increases and the capacitance of the humidity sensor will increase too. The sensor operates based on the equation of a simple parallel plate capacitor

$$
c=\frac{\varepsilon_{0} \varepsilon_{r} A}{d}
$$


Where $C$ is the capacitance of the sensor, $\varepsilon_{0}$ is the permittivity of vacuum, $\varepsilon_{r}$ is the relative permittivity of the dielectric material, $\mathrm{A}$ is the cross-sectional area of the metal plate and $d$ is the distance between the two conductive parallel plates.

\subsubsection{Design}

For temperature sensor, serpentine resistive electrodes structure was utilized .For humidity sensor, an interdigitated electrodes structure was used.

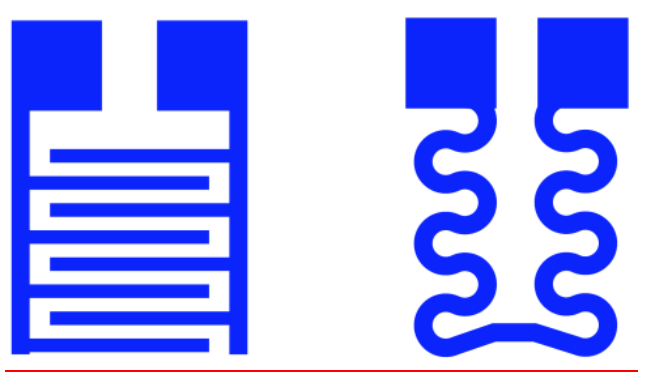

Figure 2.13: Sensors design. humidity sensor (left) and temperature sensor (right)

\subsubsection{Materials}

Copper was used as the conductive material of the sensor. The electrical resistivity of copper is $1.68 \times 10^{-8} \Omega m^{[58]}$ and The theoretical TCR of copper at $20^{\circ} \mathrm{C}$ is $.0039 \mathrm{C}^{-1}$ [57]. Copper was chosen as the conductive material of the sensor since copper has good conductivity, good flexibility and it is cost-effective compared to other widely used metals. Polyimide is used as the substrate material because of its cost-effectiveness, good long-term stability, its high sensitivity to humidity and to its compatibility with other microfabrication processes ${ }^{[51]}$. 


\subsubsection{Fabrication Process}

The same fabrication process that was used to fabricate the heater layer was used here to fabricate the sensors since it is a rapid cost-effective method and it would allow for the fabrication of both the microheaters and the sensors on the same layer. The sensors were fabricated by electroplating $8 \mu \mathrm{m}$ of copper on a on $25 \mu \mathrm{m}$ thick polyimide film (Good Fellows). Electroplating was chosen in this case as it is more efficient in terms of time than sputtering. The time needed to electroplate $8 \mu \mathrm{m}$ of copper is 45 minutes using current of .210 A. The prior to electroplating, the surface of polyimide substrate was treated with oxygen plasma to activate the surface and improve adhesion. After that and thing gold $150 \mathrm{~nm}$ layer was deposited using sputtering to act as a seed or adhesion layer for

electroplating. After that, the sensors design/structures were pattern by laser ablation method using $1.06 \mu \mathrm{m}$ ytterbium-doped fiber laser (PLS6MW Multi-Wavelength Laser Platform, Universal Laser Systems). The laser settings that were optimized for the pattering recipe are $30 \%$ power, $40 \%$ speed of the laser total capability.

\subsection{Device Integration}

\subsubsection{Bonding Process}

The expandable composite layer was spin coated on top of the heater layer and cured in the oven at $70{ }^{\circ} \mathrm{C}$ for 2 hours. Spin coating the composite layer instead of bonding it with an adhesive tape provides more reliable and stable contact between the heater and the expandable polymer. After that, the reservoir layer was bonded on top of the expandable 
composite layer using double sided adhesive film(30 $\mu \mathrm{m}$ thick, ARclear, Adhesive Research) allowing for direct contact between the expandable polymer and the reservoirs. The liquid reservoirs were filled with food dye colored water though predefined holes in the structure. The filling was achieve using a fine needle and then the holes were sealed with transparent, water-proof tape (3M Corporation). A final thin layer of PDMS was spun coated for total encapsulation. The inner surface of the microfluidics PDMS layer was coated with thin layer of parylene $\mathrm{c}$ to prevent the evaporation of liquid through preamble PDMS. To complete the system, a driver circuitry was constructed to provide sufficient current for all heating elements to reach a certain temperature (above $85 \stackrel{\circ}{\circ})$. A $3.7 \mathrm{~V}$ lithium battery was used the power unit and Adafruit Trinket mini microcontroller was used as the processing unit. SHT31x on flex humidity and temperature sensor were used as well. Figure 2.14 shows schemtic illustration of the different layers composing the device.

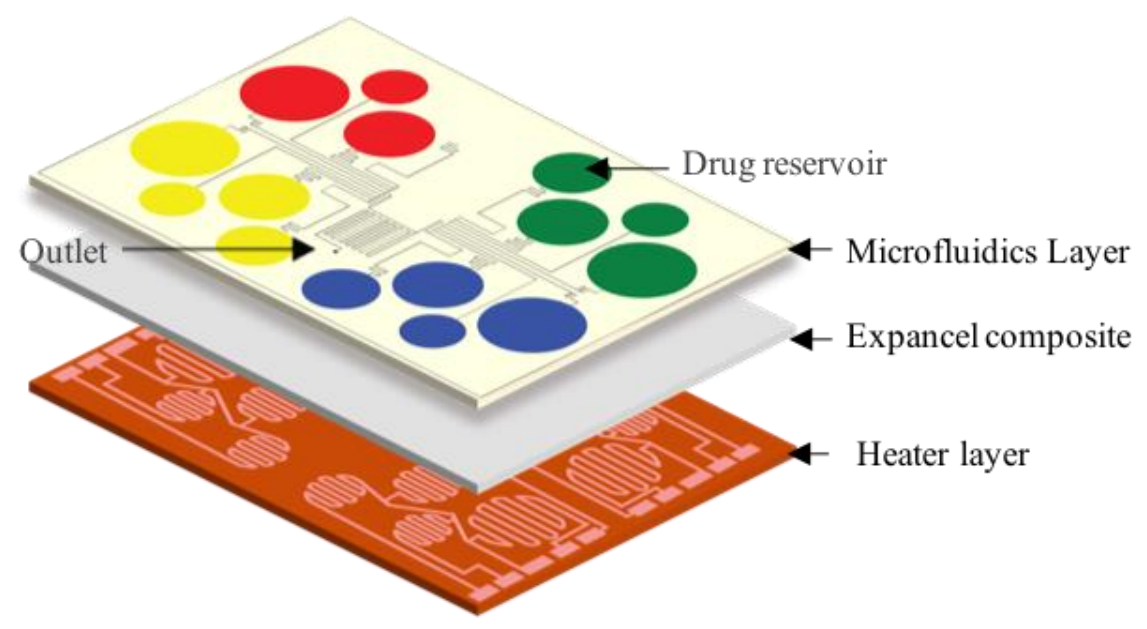

Figure 2.14: Exploded view schematic illustration of device. 


\subsubsection{Microneedles integration}

A stainless-steel hollow microneedle purchased from Micropoint Technologies (Micropoint Technologies Pte Ltd, Singapore) was integrated into the microfluidics chip using clear epoxy resin. The microneedle was placed underneath the outlet at the end of the mixing structure. Stainless steel is the most commonly used material for needles in the medical industry. It has many advantages including its low cost, biocompatibility, durability, scalability and mechanical robustness. It was chosen to integrate stainless steel hollow microneedles into the flexible PDMS substrate such that the stainless-steel needles offer the sharpness and the mechanical stability needed to penetrate the skin while the soft elastomeric PDMS base allows for the conformal interface between the microneedle and the curvilinear architecture of the skin ${ }^{[59]}$.

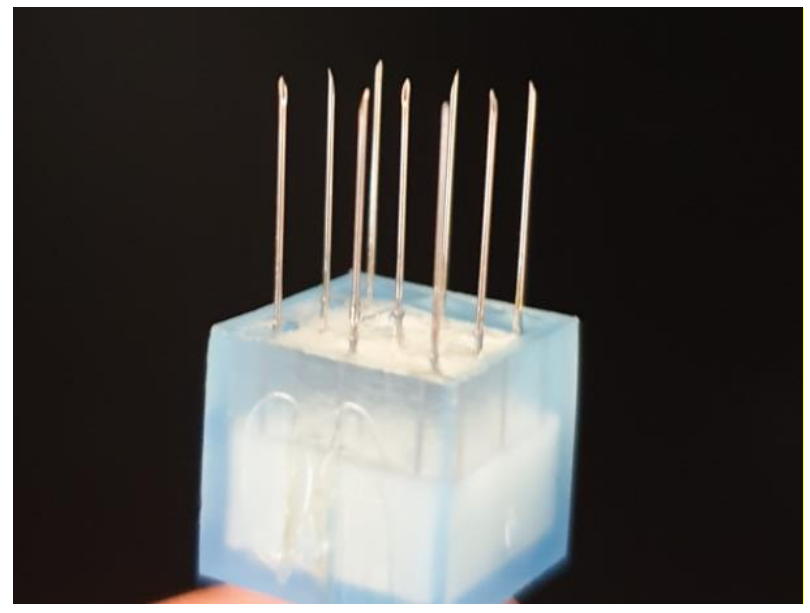

Figure 2.15: Optical photograph of Micropoint stainless-steel microneedle array 


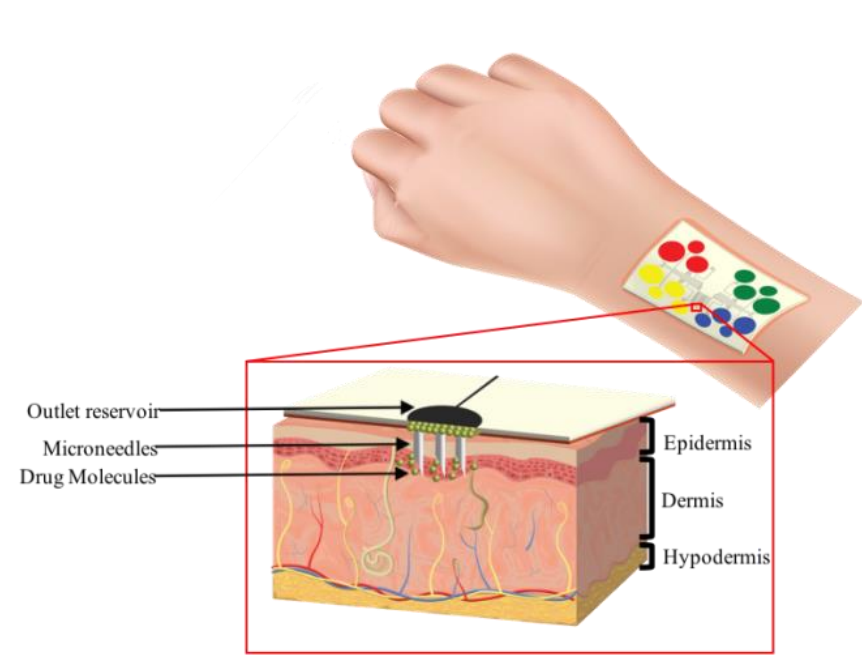

Figure 2.16: Schematic of microneedle integration into the device as well as the penetration of the needles though the skin layers

\subsubsection{Device disposability and drug re-fill}

The platform is designed in a way such that the reservoir filled layer is disposable. This approach reduces the complexity of the device and is appropriate with the irreversible nature of the actuation unit being used. The upper microfluidics layer is the only disposable layer in the system as demonstrated in figure 2.17a. After depletion of all the reservoirs on the chip, the user can easily peel off, dispose and replace the old chip with a new filled one of prescribed drugs and medications as seen in figure $2.17 \mathrm{~b}$. This method is also convenient for the user since replacing the old depleted containers is easier than requiring the user to fill them like other conventical methods. The stainlesssteel needle used has a height of $800 \mu \mathrm{m}$, the tip is less than $1 \mu \mathrm{m}$, outer diameter is $250 \mu \mathrm{m}$ while the inner diameter is $150 \mu \mathrm{m}$. The needle is strong enough for repeated skin penetrations. The height of the microneedle is long enough to penetrate the upper 
layer of the skin while it is short enough to not reach the nerve endings in the lower layer of the skin, thus, providing pain-free experience during drug administration.

a)
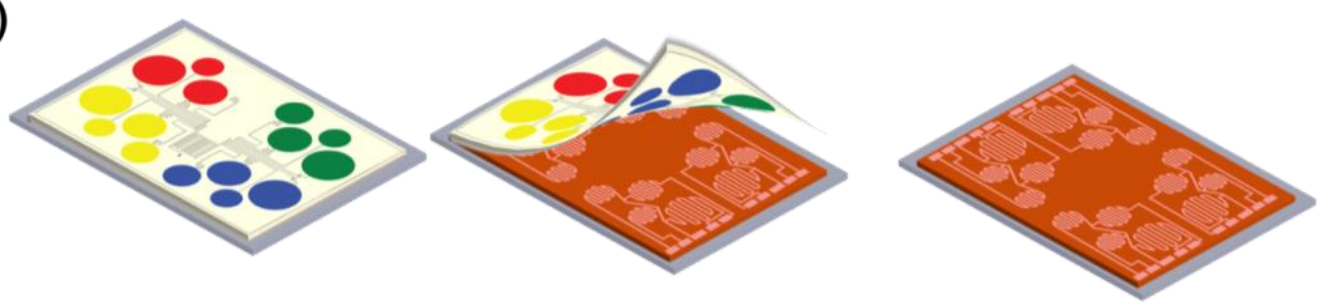

b)

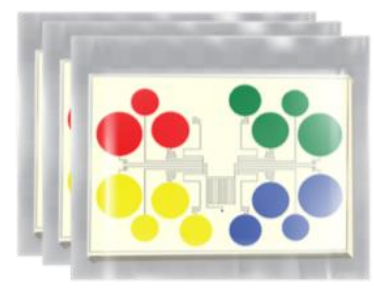

Figure 2.17: Device disposability and re-fill: a) The process of peeling off the disposable layer of the device. b) Example of filled and sealed reservoir patches fo re-fill. 


\section{Chapter 3}

\section{Results and Discussion}

\subsection{Heater Characterization \& Optimization}

\subsubsection{Heater Geometry Optimization}

Since the expansion of expancel composite strongly depends on temperature, the three chosen heater design patterns were investigated regarding the maximum temperature, power consumption and temperature distribution covering the heater area. The objective was to find the optimal pattern that will provide the required critical temperature of 80 ${ }^{\circ} \mathrm{C}$, consumes the least amount of power and provides the best temperature distribution over the heating area among other designs.

To test the functionality of the heater, a power supply was used to provided different actuation voltages of $1 \mathrm{~V}, 1.25 \mathrm{~V}, 1.5 \mathrm{~V}, 1.75 \mathrm{~V}$ and $2 \mathrm{~V}$ with a heater on time of 5 seconds. The potential applied at the 2 pads of the heater causes current to pass through the heater resulting in Joule heating. The rise in temperature and mapping of the heating profile was captured using infrared thermal imaging camera. Although many tests were perfumed for various temperatures, for power analysis purposes, only the power consumed when the maximum temperature was reached is close to the critical temperature of around $80{ }^{\circ} \mathrm{C}$ was reported. The result of thermal analysis performed of the three patterns are tabulated in table 3.1. Based on power consumption, the spiral design was found to be better compared to the meander and fan designs consuming 675 
$\mathrm{mW}$ which is around $23 \%$ less than power consumed by the fan -shaped heater and $45 \%$ less than the meander-shaped heater. Moreover, regarding the heat distribution, the average temperature was recorded. The spiral design also provided better heat distribution than the meander and fan designs with average temperature of $52.1^{\circ} \mathrm{C}$ compared to $48.9^{\circ} \mathrm{C}$ and $43.41{ }^{\circ} \mathrm{C}$ for the fan and meander designs respectively. Thus, the spiral pattern was selected as the optimal design to be used for the microheaters in this device. The spiral design provided maximum temperature of 81.25 which is within the requirement of the heater's temperature. In addition, it provided more uniform heating. Hence, an actuation voltage of $1.25 \mathrm{~V}$ with a heater on-time of 5 seconds will be used for the spiral heater.

Table 3.1: Thermal Analysis of microheater patterns

\begin{tabular}{|c|c|c|c|c|}
\hline Heater Design & $\begin{array}{c}\text { Maximum } \\
\text { Temperature } \\
\text { (ㅇ) }\end{array}$ & $\begin{array}{c}\text { Average } \\
\text { Temperature } \\
\text { (이) }\end{array}$ & $\begin{array}{l}\text { Power } \\
(\mathrm{mW})\end{array}$ & $\begin{array}{c}\text { Thermal } \\
\text { Image }\end{array}$ \\
\hline Meander & 83.2 & 43.41 & 1230 & \\
\hline Spiral & 81.25 & 52.1 & 675 & \\
\hline Fan & 84.2 & 48.9 & 878 & \\
\hline Coil & 81.9 & 55.36 & 620 & \\
\hline
\end{tabular}


In order to investigate the reasoning behind why the spiral design performed better than the other, one needed to take a closer at the effect of the geometrical elements present in each shape. One critical geometrical element is the number of corners or sharp edges the pattern contains. Sharp corners cause electron accumulation and current crowding. This electron accumulation leads to the generation of microcracking, electromigration failure and localized heat rise which all result in a shorter lifetime of the heater and lower maximum temperature it can reach. The results of several studies that investigated the performance of different heater geometries confirm the results found here ${ }^{[60][61]}$. Thus, minimizing the number of corners and having rounded coroners was reported to yield better power efficiency as the electron accumulation is reduced, the localized temperature rise is reduced resulting in an overall increase in the maximum temperature reached by the heater ${ }^{[60]}[61]$. An additional geometry was added to the thermal analysis, which is a circular spiral pattern or coil pattern. Based on the discussion mentioned above, it is expected that circular spiral heater will be more power efficient due to the absence of sharp corners and edges and minimization of heat losses. The result of the circular spiral heater analysis can be found in table 3.1. Indeed, the coil spiral pattern reached the critical temperature of $81.9^{\circ} \mathrm{C}$ with less power applied compared to the square spiral design. Although this difference in power consumption might be considered small, the coil spiral design also provides better heat distribution with an average temperature of $55.36^{\circ} \mathrm{C}$. Thus, it was determined that the circular/coil spiral design will be used as the pattern for the microheaters of the device. 


\subsubsection{Heater Characterization}

Figure 3.1 shows the temperature rise over time after turning the heater on. It can be seen that initially the heater experiences a rapid temperature rise reaching the critical temperature of $>80^{\circ} \mathrm{C}$ after 1.5 seconds only. After that, the increase in temperature slows down and almost levels off after 2.5 seconds. Figure 2.1 shows IR thermal images of a single heater powered on or multiple heaters at the same time. The IR images shows the isolated Joule heating for each heater confirming the functionality of the design.

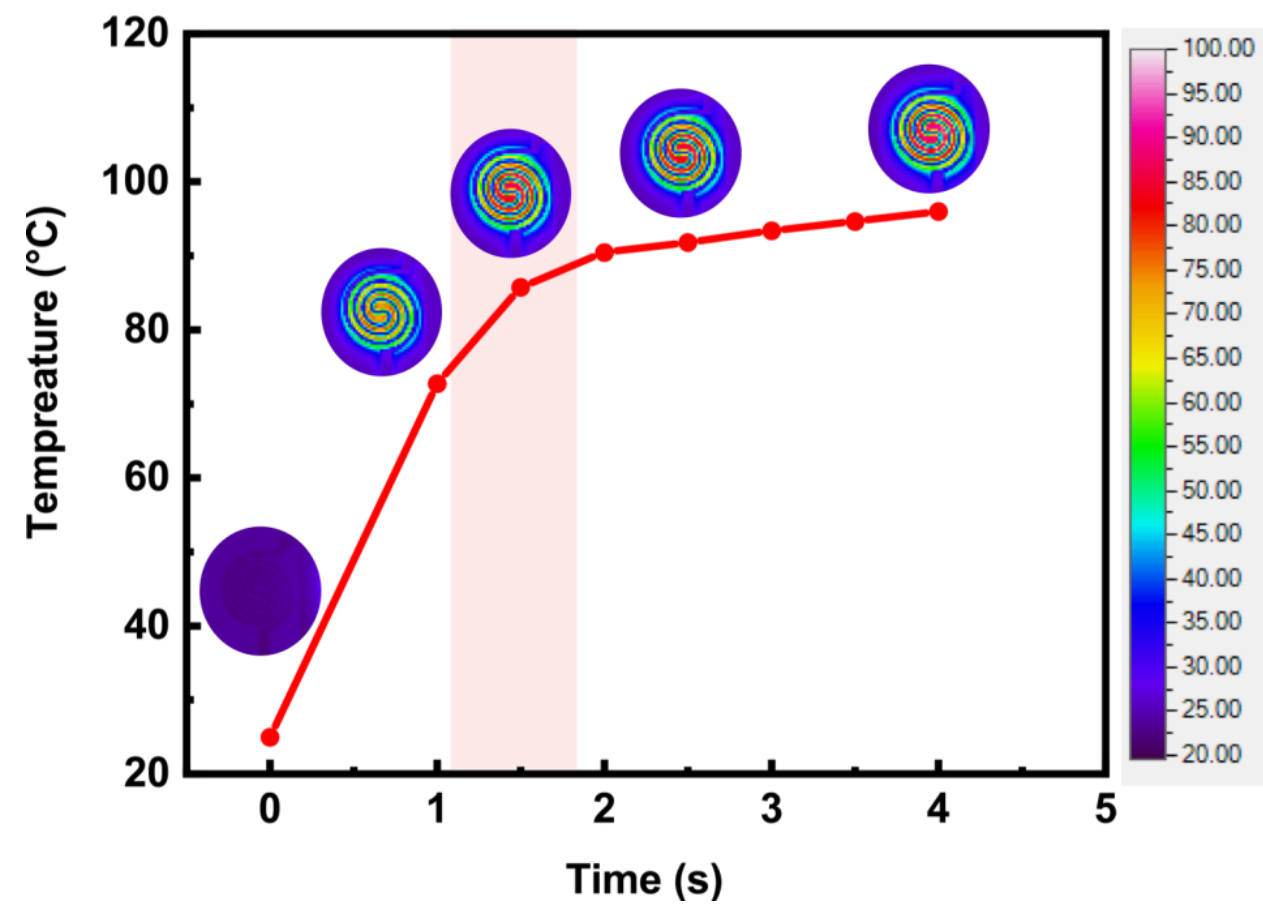

Figure 3.1: Heater temporal Characterization. Experimental results of temperature increase upon triggering the heater showing a period of 1.5 seconds needed to reach the critical temperature of $>$ $80^{\circ} \mathrm{C}$. 

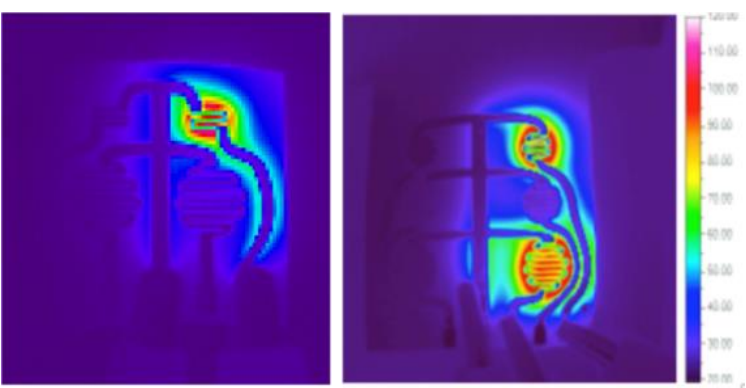

Figure 3.2: Thermal characterization of microheaters. Infrared (IR) thermal images of powering of single heater (left) and multiple heaters at simultaneously (right).

a)
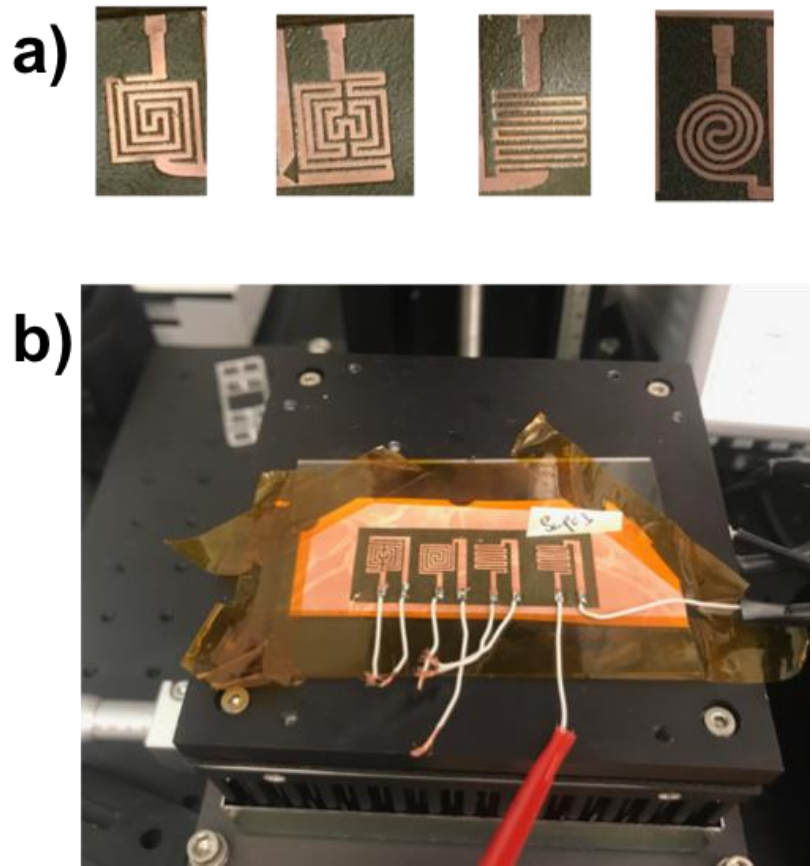

Figure 3.3: Heater testing setup. a) Optical photographs of the four different patterns of the fabricated heaters. b) Experimental setup for heater thermal characterization using infrared (IR)

\subsection{Actuator Unit Characterization \& Optimization}

In order to characterize the thermal expansion behavior of the expandable composite, layer of the expandable composite was placed on top of the heater by spin coating and curing. Figure 3.4a below shows an optical photograph of the side view of the expansion 
profile after heater activation while Figure $3.4 \mathrm{~b}$ shows SEM images of expancel ${ }^{\circledR}$.

Figure3.5 shows SEM images of expancel microspheres before and after heater activation.

It can be seen that on average, the microspheres experienced enlargement of up to two and a half times its original size.

a)

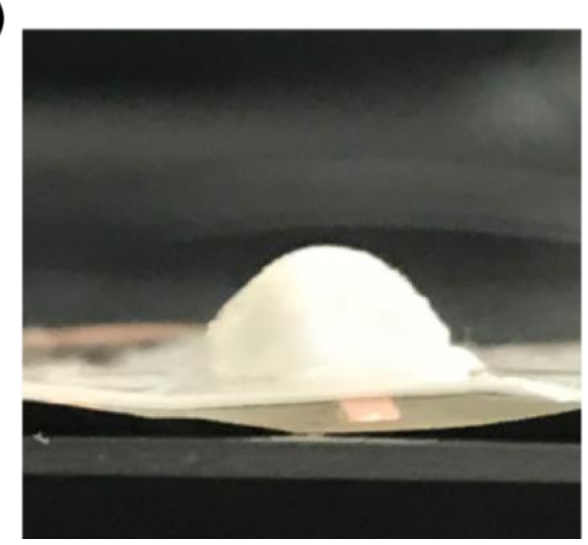

b)

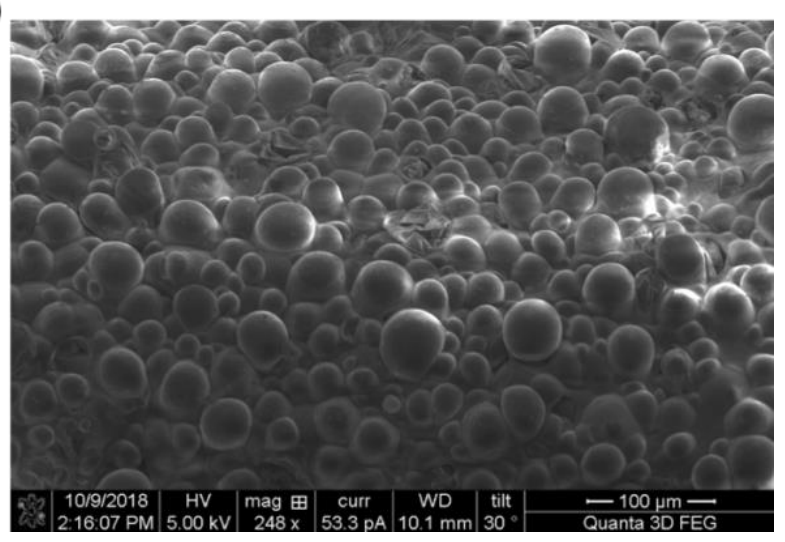

Figure 3.4: Expancel Charactrization. a) Optical photograph showing the side view of the expansion profile of the composite. b) SEM images of Expancel ${ }^{\circledR}$ microspheres in their expanded
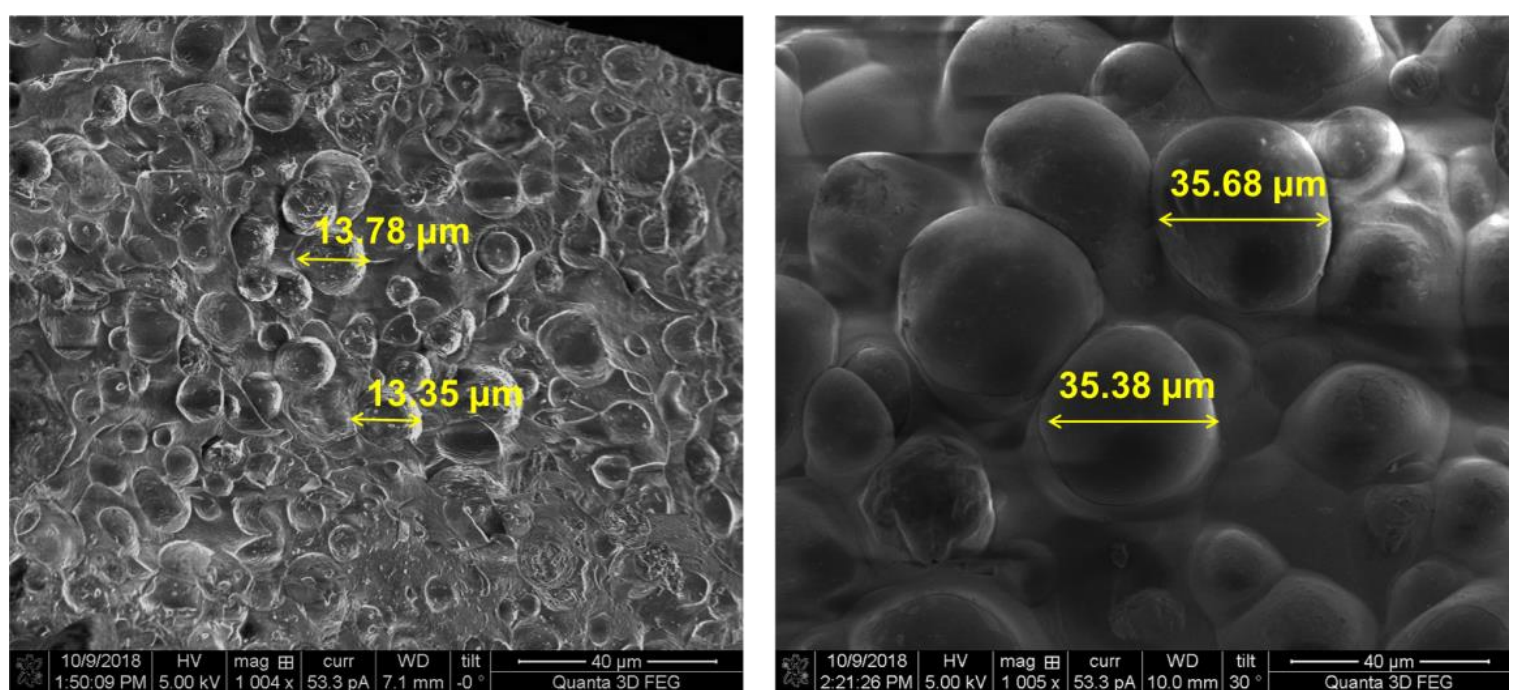

Figure 3.5: SEM images of Expancel ${ }^{\circledR}$ microspheres unexpanded microspheres (left) and fully expanded microspheres (right). 


\subsubsection{Expancel-PDMS layer thickness optimization}

The thickness of the PDMS-expancel composite is an important parameter that affect both the expansion ratio as well as the amount of fluid being infused from the drug. The goal is to deplete the reservoir completely in order to deliver the precise amount of desired dosage that was predefined by the reservoir volume. Thus, the optimum thickness of PDMS-expancel composite layer was investigated by altering the spin coating speed during fabrication. Figure3.6 shows the different thicknesses that were used and the corresponding amount of drug volume that was infused for each thickness used in the experiment. The amount of drug volume infused is represented as a percentage from the original amount of drug in the reservoir. The experimental results showed that the optimal thickness of PDMS-expancel composite is around $280 \mu \mathrm{m}$, which infuses $93.33 \%$ of the total volume of the reservoir.

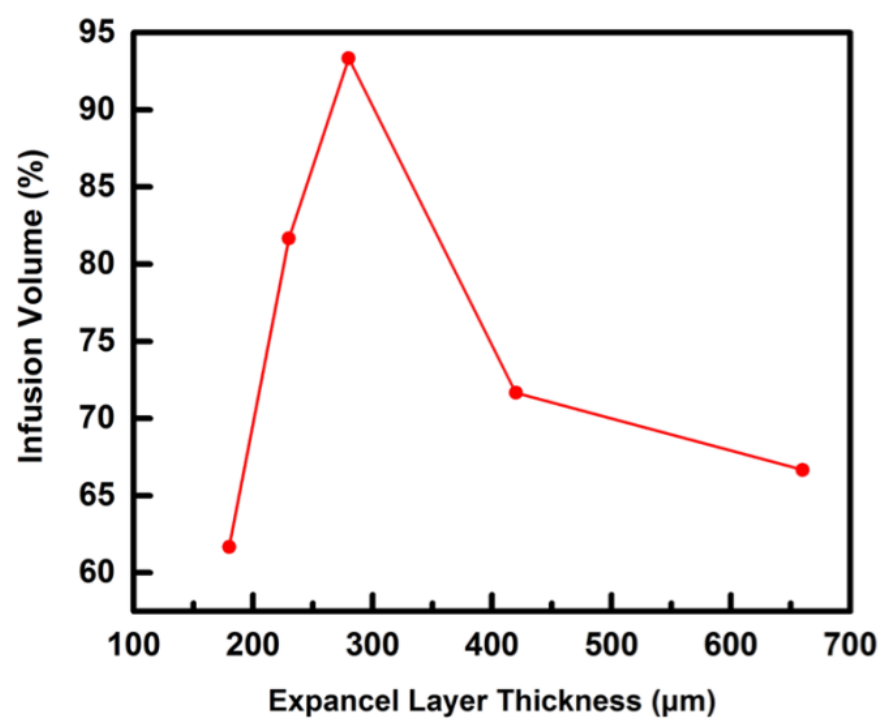

Figure 3.6: Drug infusion volume as a function of thickness variation of expancel composite 
It can be seen from figure 3.6 above that the relationship between the thickness of expancel layer and the infusion volume starts as an almost linear relationship which is expected. The thicker the layer is, the more expancel microspheres are there ready to expand and as a result the total effect of expansion of each is reflected in an overall larger expansion profile. Better and higher volume expansion corresponds to the ability of the expanded layer to cover majority of the reservoir volume and thus displacing most of the fluid out of the reservoir. Beyond $280 \mu \mathrm{m}$, however, it was observed that as the thickness of expancel layer increases, the amount of drug being infused decreases. This can be explained by looking at the thermodynamic behavior of expancel and its properties in the expanded state. Expancel microspheres in their expended state act as good thermal insulating unit and has been used as thermal insulating coating for many applications including cables filling, roofs and walls of houses ${ }^{[47]}$. This is because when it is expanded, the gas-filled voids in the composite and the phase change decreases the thermal conductivity of the overall composite from .2 to $0.06 \mathrm{~W} / \mathrm{m}^{*} \mathrm{~K}^{[47]}$. It is reported that this thermal insulation combabilities can be observed with layers of thickness of around 1.0 to $1.5 \mathrm{~mm}$ for optimal thermal insulation performance ${ }^{[47]}$. Taking another look at the graph in figure 3.6, it can be noticed that as expancel layer becomes thicker, the amount of drug infused decreases which implies that expancel layer didn't provide sufficient expansion to deplete the reservoir. This indicates that at thickness above $280 \mu \mathrm{m}$, expansion of expancel is slowed down as the expanded microspheres that located close to the heater are now acting as thermal insulating layer for the upper unexpanded microspheres $^{[52]}$. This will overall halt the expansion and decreases it. Thus, the behavior 
of expancel composite can be divided into two main regions as demonstrated in figure 3.7. The first region is when the overall expansion is limited by the number of microspheres that exist, which representative in the thickness of the composite. This can be considered as the ideal thermodynamic expansion behavior of expancel. In this region, thickness of the composite layer and the amount of drug being infused is almost linear. In the second region which lies beyond the thickness of $280 \mu \mathrm{m}$, the overall expansion is limited by the heat transfer as the already expanded Expancel layer acts as thermal insulating unit which makes it difficult for heat to be conducted and reach the upper unexpanded part of the layer. This decline of expansion leads to an overall expansion height that is less than the ideal expanded height, which results in less volume being displaced from the reservoir.

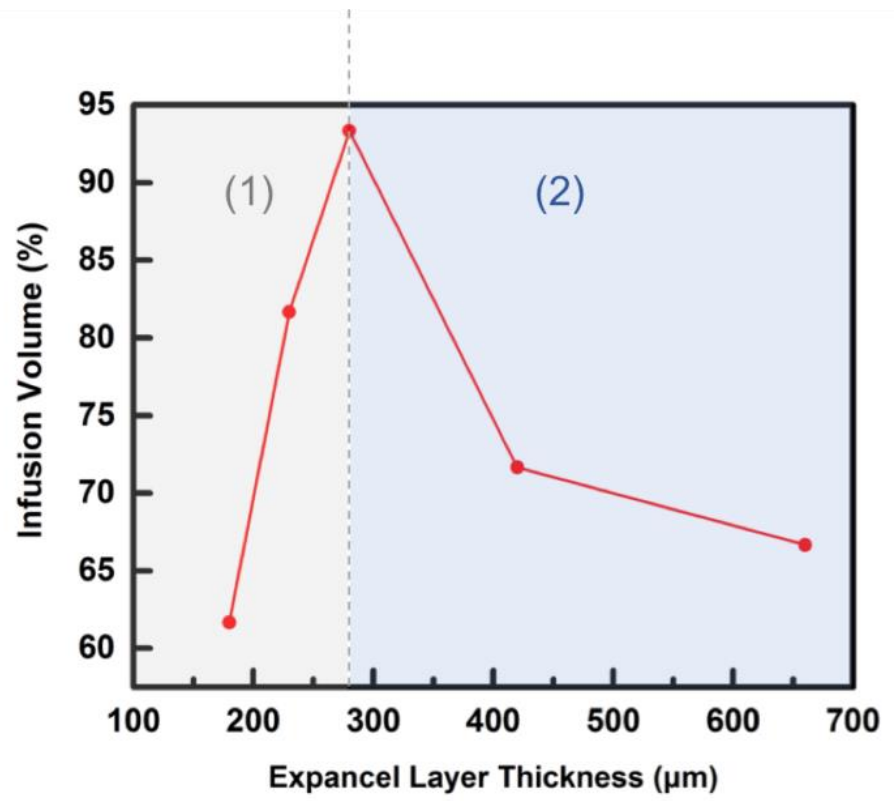

Figure 3.7: A Drug infusion volume versus expancel composite thickness showing the two main regions of expansion behavior. 


\subsection{Microfluidics Layer Characterization \& Optimization}

\subsubsection{Reservoir Geometry optimization}

Another important parameter that affect the volume of liquid infused is the shape of the fluidic reservoir. Different shapes of reservoir were fabricated and tested including a cylinder, a hemisphere a square and a truncated cone reservoir. The results in graph 3.8 indicate that the hemispherical type of reservoir infused the highest volume of liquid with a percentage of $\sim 95 \%$ of the original reservoir volume. This result can be explained by looking at the molecular shape of expancel. Expancel consist of tiny microspheres that contain liquid hydrocarbon encapsulated inside a thermoplastic shell. When heated, the thermoplastic shell softens and the enclosed hydrocarbon changes phase from liquid to gas and results in expansion. Upon expansion, the microspheres still maintain their spherical shape and the overall expansion profile of the composite forms an almost hemispherical shape that can see in $3.9 \mathrm{~b}$. This expansion profile conforms the most to the hemispherical reservoirs, and as a result it depletes most of the liquid out of the reservoir more efficiently than the other shapes. 


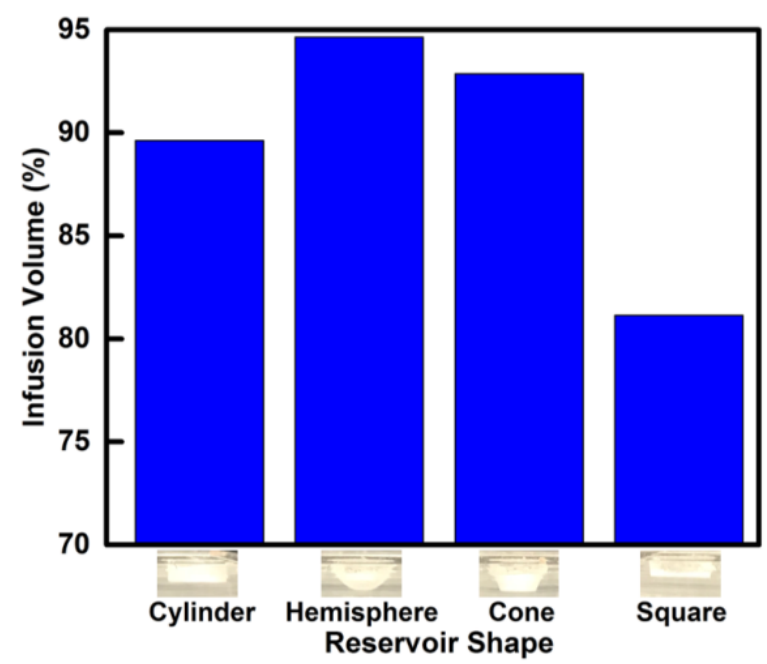

Figure 3.8: Drug infusion volume as function of the reservoir shape/geometry

a)

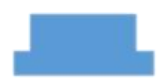

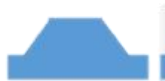
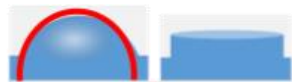

b)

Figure 3.9: Reservoir Geometry conformity. a) Schematic designs of the four reservoir geometries used. b) Optical photograph showing a side view of the hemispherical expansion profile.

\subsubsection{Microfluidics Mixing}

\section{Micro Mixer}

Adding mixing capability to the platform is a first step to enable formulation and preparation of drug microcarriers in the future. For demonstration and proof of concept purposes, food dye colored water solutions were used and filled up the reservoirs. By selectively triggering different drug reservoirs of the platform, the desired fluids were ejected out of the device as seen in figure 2.10. Initially, reservoir 1 was filled with green colored water and reservoir 2 contained red colored water shown in figure 3.10b. Upon 
triggering the two heaters simultaneously, blue and yellow solutions were ejecting from their prospective reservoirs separately and then mixed (green and red) to form a desired mixture output (yellow) at the outlet as can be seen in Figure 3.10c below.
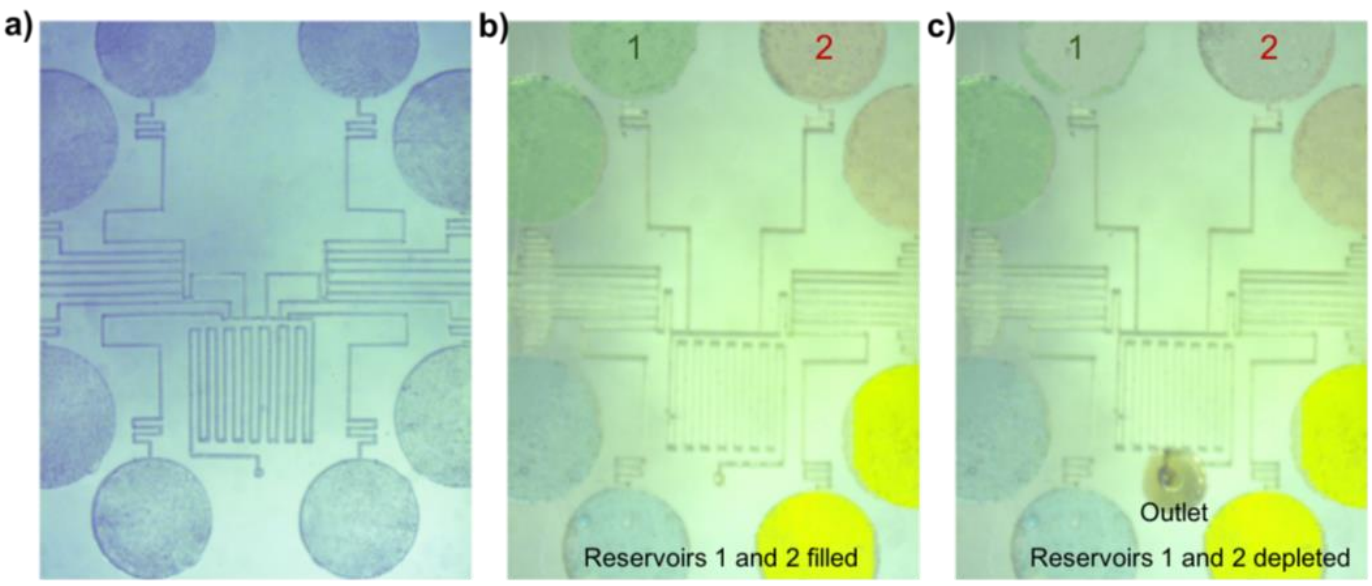

Figure 3.10: Validation of microfluidic mixing capability of the device (red and green liquid mixture yielding a yellow colored liquid

a)

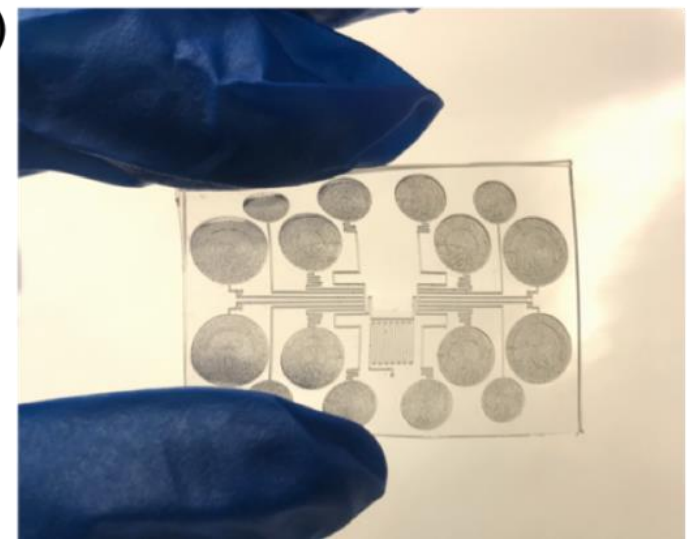

b)

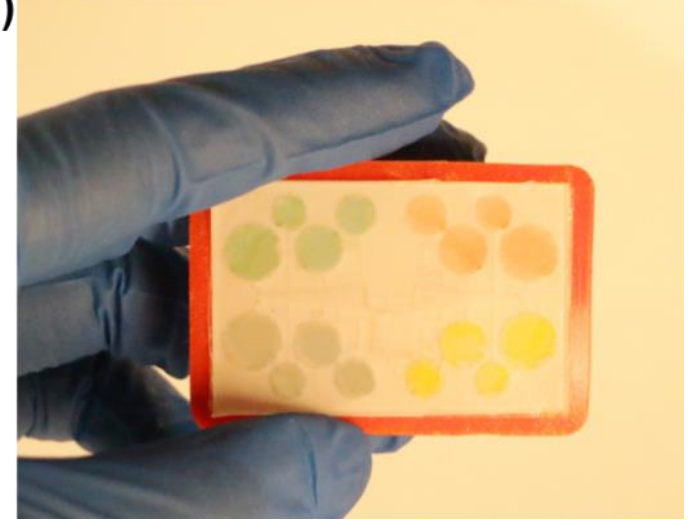

Figure 3.11: a) Photograph of the PDMS microfluids layer showing the reservoirs and micromixer. b) Photograph of the microfluidics layer after filling and sealing the reservoirs 


\subsection{Wearable Sensors}

\subsubsection{Temperature and Humidity Sensor Characterization}

In order to test the performance of the fabricated sensors, the sensors were subjected to conditions such as heating from a hot air gun for characterizing the temperature sensor and exposure of the blow of breath for characterizing the humidity sensor. The raw data and readings for both sensors were collected from Keithley 4200 parameter analyzer. In order to correlate the raw output of resistance and capacitance from Keithley to its corresponding temperature value and humidity percentage, the system was calibrated using a commercial humidity/temperature sensor (sensirion ${ }^{\circledR}$ Digital Humidity and temperature Sensor SHT3x) which was used at the same time of preforming the experiment. Figure 3.12 shows optical photographs of the fabricated humidity and temperature sensors. The performance of the temperature sensor is almost linear with sensitivity of $33.8 \mathrm{~m} \Omega /{ }^{\circ} \mathrm{C}$ over a range of temperature between 20 to $60{ }^{\circ} \mathrm{C}$ as seen in the graph of figure 3.12. The characterization graph of humidity sensor is shown in figure 3.14 where the sensor was subjected to three alternating cycles of human breath. The graph demonstrates the real-time reliable response and recovery behavior of the fabricated sensor and its consistency with the performance of state-ofart commercial sensing standards. 


\section{3}
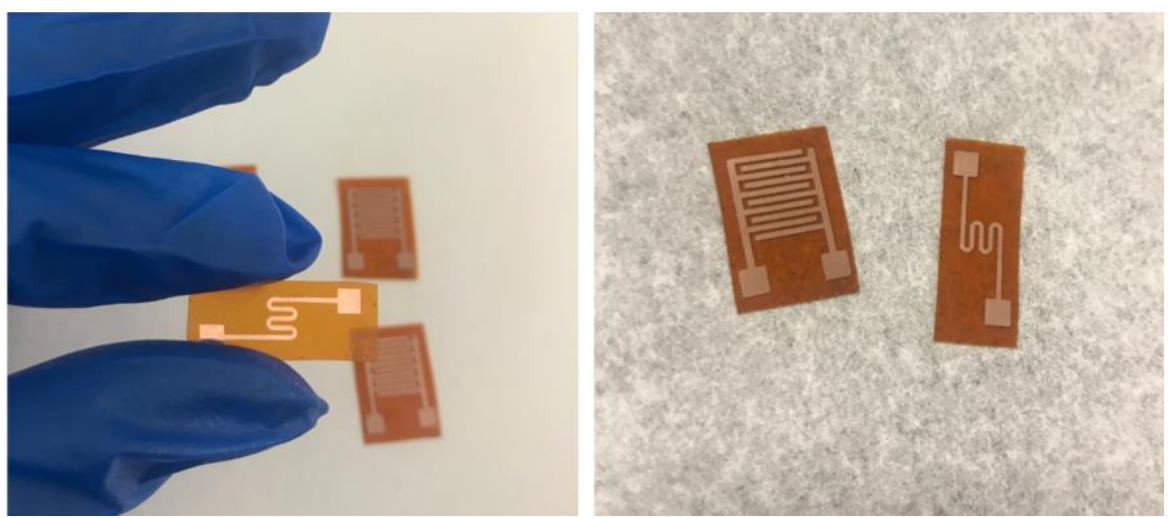

Figure 3.12: Optical photographs of the fabricated temperature resistive sensor and capacitive-based interdigitated humidity sensor made out of copper and PI.

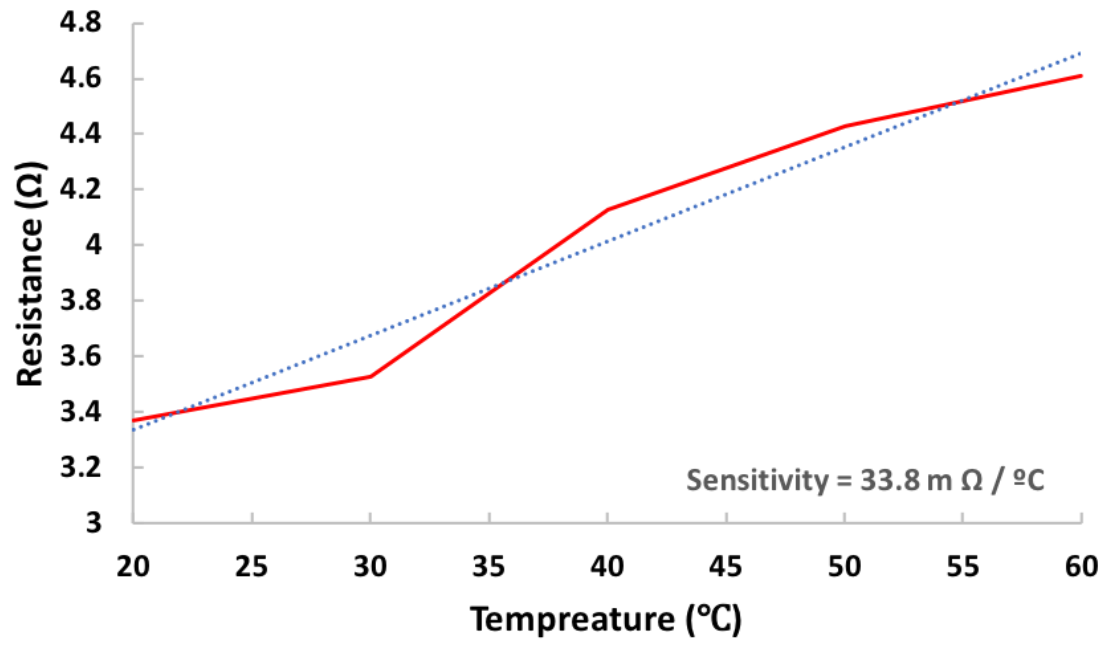

Figure 3.13: Temperature sensor characterization plot of resistance versus temperature. 


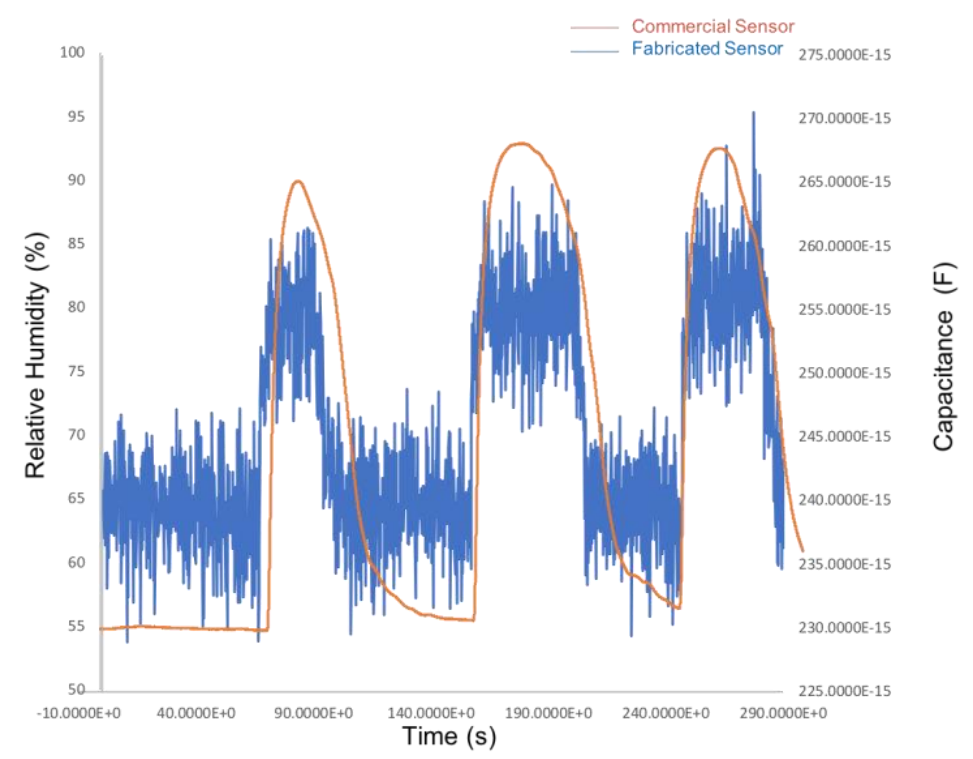

Figure 3.14: Humidity sensor characterization plot of capacitance versus relative humidity (when exposed to alternating intervals of human breath.

\subsection{System Integration}

To assess the fluid release through the microneedle, a layer of Ecoflex ${ }^{\circledR}$ 00-30 silicone elastomer was used to mimic the skin. Figure 3.15a shows optical photographs of the microneedle when integrated into the platform. Figure 3.15b shows the successful penetration of the microneedle into the skin mimicking layer as well as the successful infusion of liquid into the layer. 
a)
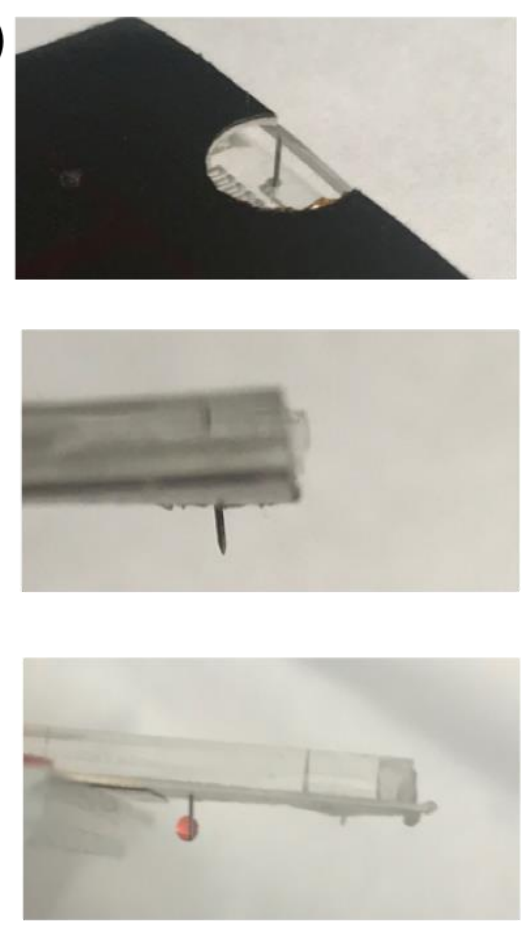

b)
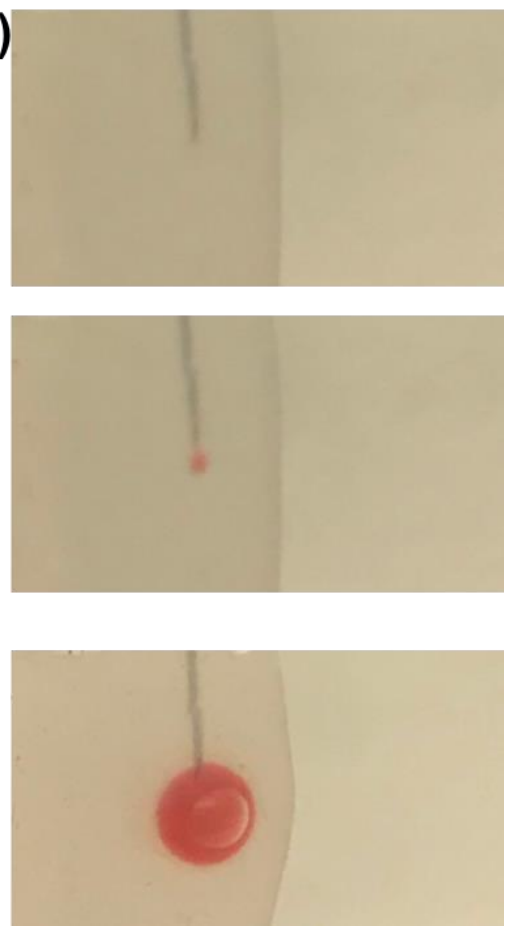

Figure 3.15: Microneedle integration and testing. a) Optical photographs of the microneedle integrated into the platform. b) Sequence of photographs showing the successful insertion of microneedle into the phantom skin as well as liquid delivery.

In order to integrate the platform into complete system, electronic components such as microcontroller, current driver circuit and a battery were used. Figure3.16 is a photograph that shows the different components integrated into the platform. Adafruit Trinket microcontroller is used to program the selective actuation of each heater. The current driver circuit is used to control and provide sufficient current for all heating elements to reach the actuation temperature of $>80 \mathrm{C}$. A $3.7 \mathrm{~V}$ lithium battery was used to provide power to the system. The heater and circuit layer maintain their flexibly even after soldering the electronic components, which provide the needed conformability for such wearable device to be fully functional and comfortable for a long period of time. 
a)

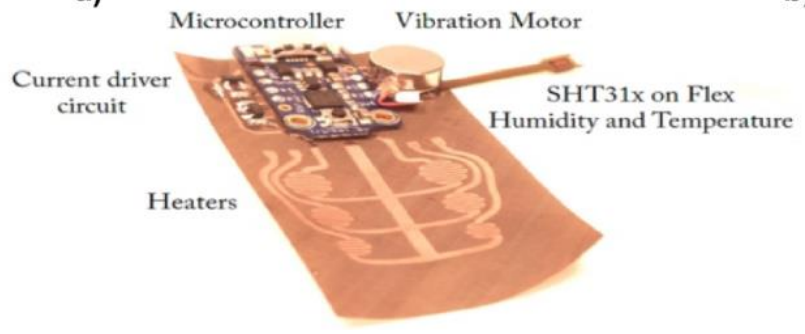

b)

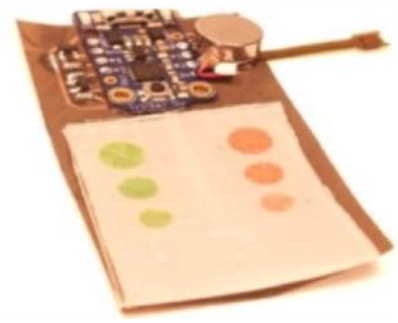

Figure 3.16: Photographs of the integrated system.a) and b) Integrated system for wearable transdermal drug delivery platform

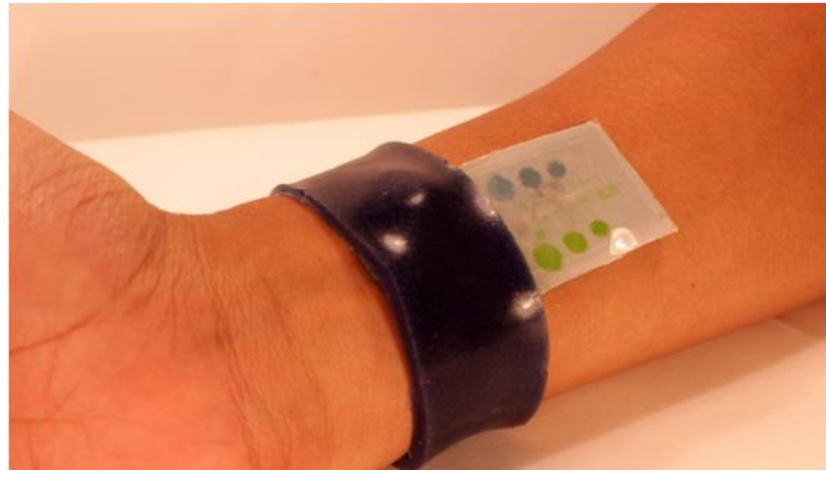

Figure 3.17: Photograph of the wearable platform on a wrist

a)

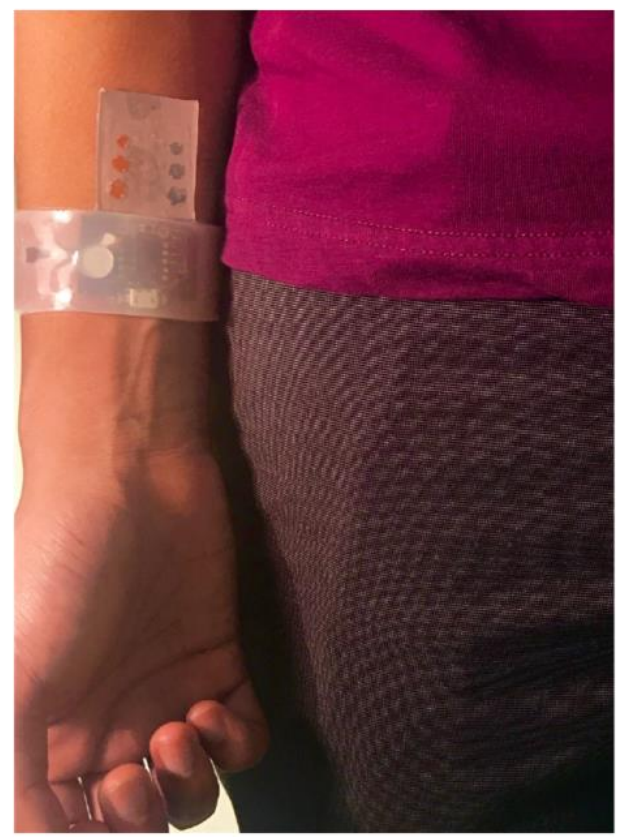

b)

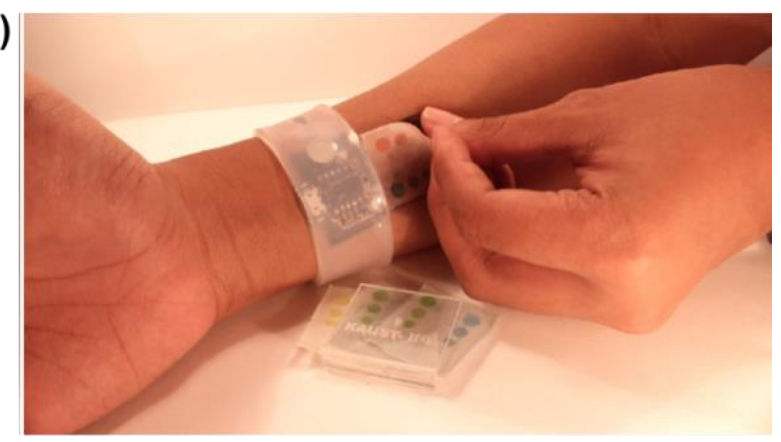

c)

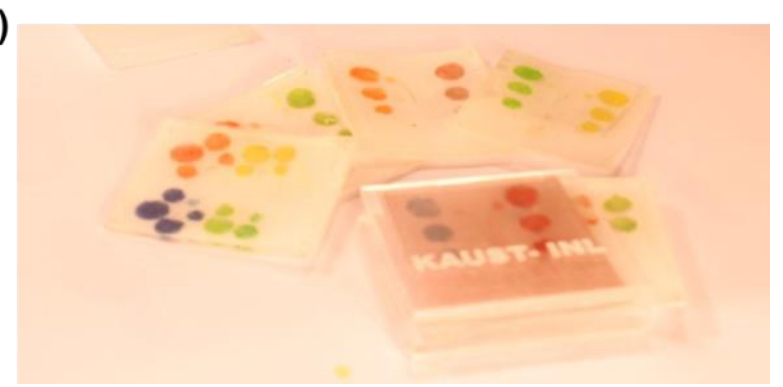

Figure 3.18: a) Disposability of the wearable platform b) a photograph showing the mechanism of disposability of the device by peeling off the upper microfluidics layer. c) Sample of filled reservoirs layers for replacements 


\subsubsection{Closed-Loop Experiment}

In order to demonstrate the application of the integrated system in a closed-loop feedback autonomous manner, the platform was placed on the wrist of a subject during physical exercise. After real-time detection of physical fatigue from wearable sensors, the corresponding programmed reservoirs were triggered to release the drug. Figure 3.18a shows the percentage of relative humidity over time. Once the programmed threshold of $95 \%$ relative humidity was reached, instant triggering of both heaters was initiated. As a result, the fluid was ejected from both reservoirs and mixed before reaching the outlet as seen in figure $3.18 \mathrm{c}$ and $3.18 \mathrm{~d}$. It is important to note here that in this experiment, and for proof of concept purpose, only the humidity and temperature sensors were used. In the future, more biosensors will be incorporated in order to provide a more accurate assessment of the physiological state of the human body.
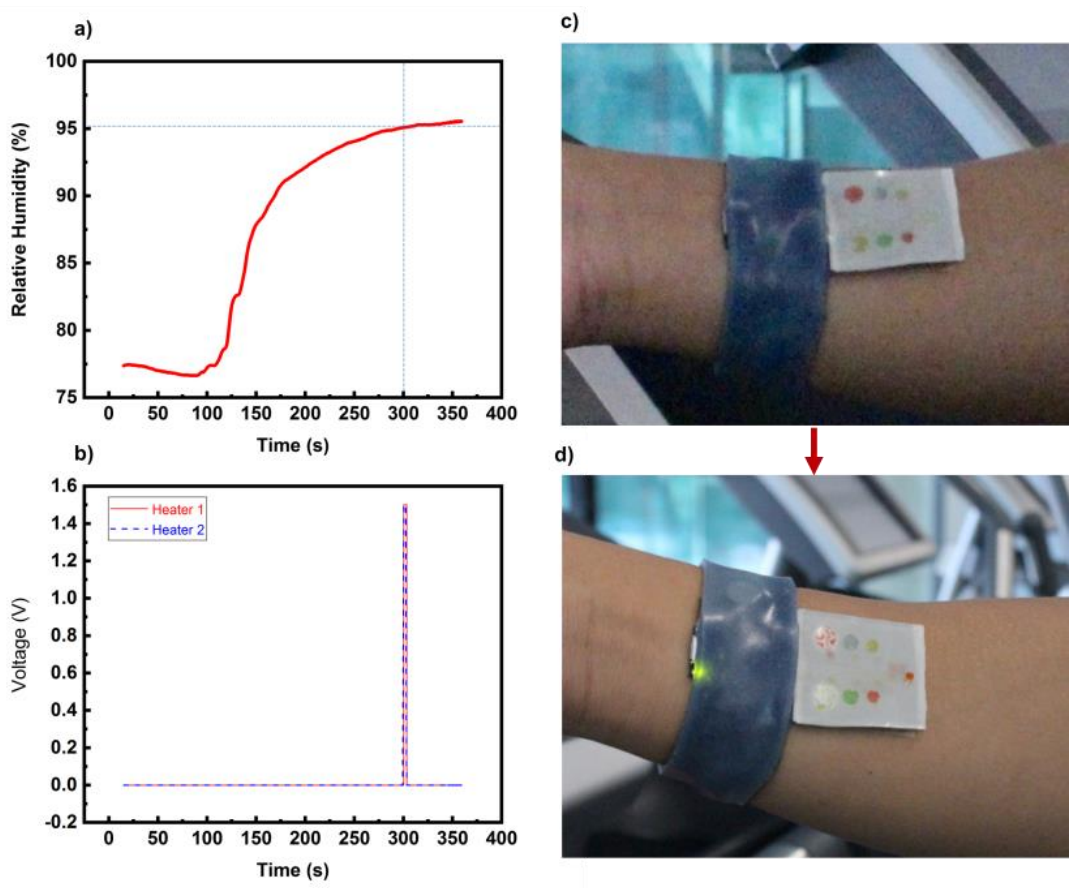

Figure 3.19: Closed-loop feedback experiment: a) and b) triggering the device after detecting physical fatigue from wearable sensors. c) the platform before and d) after actuation 


\section{Chapter 4}

\section{Concluding Remarks and Future Work}

In this project, we have shown a wearable transdermal drug delivery platform that is aimed to pursue the goal of on-demand personalized medicine. The platform successfully demonstrated

drug mixing and delivery on demand using an expandable composite as the main pumping mechanism to displace drug from pre-defined reservoirs upon triggering heating elements underneath the expandable composite. Although expancel composite have been used previously by other groups to actuate microfluidics devices, this study presented extensive optimization of important parameters in order to maximize the performance of expancel as a drug delivery pump. Rapid inexpensive fabrication approaches such as $3 \mathrm{~d}$ printing and laser micromachining were utilized in the fabrication process of the platform to reduce cost and enable its large-scale use. We have also shown the integration of wearable sensors that are continuously collecting physiological signals from the body in order to provide autonomous closed-loop therapeutic actions. This feedback function will be the next paradigm shift for healthcare in the future. Effort is needed in the future with regard to the development and packaging of the platform to enhance its stability, reliability and long-term use. In the future, the microneedles that were used for transdermal delivery can also be used for biosensing purposes. The same pump can be designed to work in a reverse manner to draw collect bodily fluid like the sweat which carries many important metabolites and analytes. Moreover, sensors and artificial intelligence algorithms can be used to assess the effect of drug after delivery and provide updated feedback on the phycological state of the body. 


\section{BIBLIOGRAPHY}

[1] J. L. Jameson, D. Ph, D. L. Longo, N. Engl. J. Med. 2015, 1.

[2] B. G. E. Moore, 1975, 38.

[3] Ranjan Singh, "Wearable Electronics Market is Expected to Reach \$25.19 Billion, Globally, by 2020," can be found under https://www.alliedmarketresearch.com/press-release/wearable-electronicsmarket-is-expected-to-reach-25-19-billion-globally-by-2020-allied-marketresearch.html, n.d.

[4] J. M. Nassar, M. D. Cordero, A. T. Kutbee, M. A. Karimi, G. A. T. Sevilla, A. M. Hussain, A. Shamim, M. M. Hussain, Adv. Mater. Technol. 2016, 1, 1.

[5] T. Q. Trung, N.-E. Lee, Adv. Mater. 2016, 28, 4338.

[6] Y. Khan, A. E. Ostfeld, C. M. Lochner, A. Pierre, A. C. Arias, Adv. Mater. 2016, 28, 4373.

[7] K. Takei, W. Honda, S. Harada, T. Arie, S. Akita, Adv. Healthc. Mater. 2015, 4, 487.

[8] Q. Li, L.-N. Zhang, X.-M. Tao, X. Ding, Adv. Healthc. Mater. 2017, 6, 1601371.

[9] C. Yan, J. Wang, P. S. Lee, ACS Nano 2015, 9, 2130.

[10] W. Honda, S. Harada, T. Arie, S. Akita, K. Takei, Adv. Funct. Mater. 2014, 24, 3299.

[11] S. Harada, W. Honda, T. Arie, S. Akita, K. Takei, ACS Nano 2014, 8, 3921.

[12] H. Yang, D. Qi, Z. Liu, B. K. Chandran, T. Wang, J. Yu, X. Chen, Adv. Mater. 2016, 28, 9175.

[13] T. Q. Trung, S. Ramasundaram, B.-U. Hwang, N.-E. Lee, Adv. Mater. 2016, 28, 502.

[14] R. Bendi, V. Bhavanasi, K. Parida, V. C. Nguyen, A. Sumboja, K. Tsukagoshi, P. S. Lee, Nano Energy 2016, 26, 586.

[15] D. Kong, L. T. Le, Y. Li, J. L. Zunino, W. Lee, Langmuir 2012, 28, 13467.

[16] T. Q. Trung, S. Ramasundaram, S. W. Hong, N.-E. Lee, Adv. Funct. Mater. 2014, 24, 3438.

[17] J. Yang, D. Wei, L. Tang, X. Song, W. Luo, J. Chu, T. Gao, H. Shi, C. Du, RSC Adv. 2015, 5, 25609.

[18] R. Chad Webb, S. Krishnan, J. A. Rogers, in (Eds: J. A. Rogers, R. Ghaffari, D.-H. 
Kim), Springer International Publishing, Cham, 2016, pp. 117-132.

[19] S. R. Krishnan, T. R. Ray, A. B. Ayer, Y. Ma, P. Gutruf, K. Lee, J. Y. Lee, C. Wei, X. Feng, B. Ng, et al., Sci. Transl. Med. 2018, 10, eaat8437.

[20] W.-P. Shih, L.-C. Tsao, C.-W. Lee, M.-Y. Cheng, C. Chang, Y.-J. Yang, K.-C. Fan, Sensors 2010, 10, DOI 10.3390/s100403597.

[21] S. Y. Hong, Y. H. Lee, H. Park, S. W. Jin, Y. R. Jeong, J. Yun, I. You, G. Zi, J. S. Ha, Adv. Mater. 2016, 28, 930.

[22] A. Hanif, T. Q. Trung, S. Siddiqui, P. T. Toi, N.-E. Lee, ACS Appl. Mater. Interfaces 2018, 10, 27297.

[23] C. Wang, K. Xia, M. Zhang, M. Jian, Y. Zhang, ACS Appl. Mater. Interfaces 2017, 9, 39484.

[24] Y. Yamamoto, D. Yamamoto, M. Takada, H. Naito, T. Arie, S. Akita, K. Takei, Adv. Healthc. Mater. 2017, 6, 1700495.

[25] R. C. Webb, A. P. Bonifas, A. Behnaz, Y. Zhang, K. J. Yu, H. Cheng, M. Shi, Z. Bian, Z. Liu, Y.-S. Kim, et al., Nat. Mater. 2013, 12, 938.

[26] L. Gao, Y. Zhang, V. Malyarchuk, L. Jia, K.-I. Jang, R. Chad Webb, H. Fu, Y. Shi, G. Zhou, L. Shi, et al., Nat. Commun. 2014, 5, 4938.

[27] Y. Li, H. Shimizu, Macromolecules 2009, 42, 2587.

[28] S. Gong, W. Schwalb, Y. Wang, Y. Chen, Y. Tang, J. Si, B. Shirinzadeh, W. Cheng, Nat. Commun. 2014, 5, 3132.

[29] C. Dagdeviren, Y. Su, P. Joe, R. Yona, Y. Liu, Y.-S. Kim, Y. Huang, A. R. Damadoran, J. Xia, L. W. Martin, et al., Nat. Commun. 2014, 5, 4496.

[30] W. Gao, S. Emaminejad, H. Y. Y. Nyein, S. Challa, K. Chen, A. Peck, H. M. Fahad, H. Ota, H. Shiraki, D. Kiriya, et al., Nature 2016, 529, 509.

[31] S. Y. Oh, S. Y. Hong, Y. R. Jeong, J. Yun, H. Park, S. W. Jin, G. Lee, J. H. Oh, H. Lee, S.-S. Lee, et al., ACS Appl. Mater. Interfaces 2018, 10, 13729.

[32] D. P. Rose, M. E. Ratterman, D. K. Griffin, L. Hou, N. Kelley-Loughnane, R. R. Naik, J. A. Hagen, I. Papautsky, J. C. Heikenfeld, IEEE Trans. Biomed. Eng. 2015, 62, 1457.

[33] J. M. Nassar, K. Mishra, K. Lau, A. A. Aguirre-Pablo, M. M. Hussain, Adv. Mater. Technol. 2017, 2, 1600228.

[34] H. Marwah, T. Garg, A. K. Goyal, G. Rath, Drug Deliv. 2016, 23, 564. 
[35] I.-C. Lee, W.-M. Lin, J.-C. Shu, S.-W. Tsai, C.-H. Chen, M.-T. Tsai, J. Biomed. Mater. Res. Part A 2017, 105, 84.

[36] D. Son, J. Lee, S. Qiao, R. Ghaffari, J. Kim, J. E. Lee, C. Song, S. J. Kim, D. J. Lee, S. W. Jun, et al., Nat. Nanotechnol. 2014, 9, 397.

[37] J. Di, S. Yao, Y. Ye, Z. Cui, J. Yu, T. K. Ghosh, Y. Zhu, Z. Gu, ACS Nano 2015, 9, 9407.

[38] H. Lee, T. K. Choi, Y. B. Lee, H. R. Cho, R. Ghaffari, L. Wang, H. J. Choi, T. D. Chung, N. Lu, T. Hyeon, et al., Nat. Nanotechnol. 2016, 11, 566.

[39] G. M. Whitesides, Nature 2006, 442, 368.

[40] C. Hansen, S. R. Quake, Curr. Opin. Struct. Biol. 2003, 13, 538.

[41] C. B,ROUSSEL S, "Microfluidic Applications in the Pharmaceutical, Life Sciences, In-Vitro Diagnostic, and Medical Device Markets," can be found under http://www.yole.fr/Microfluidic_Applications_Players.aspx\#.XLZW1pMzYnU, n.d.

[42] B. Lin, Microfluidics Technologies: Technologies and Applications, 2011.

[43] J. H. Tsui, W. Lee, S. H. Pun, J. Kim, D. H. Kim, Adv. Drug Deliv. Rev. 2013, 65, 1575.

[44] Q. Wang, J. Y. Huang, H. Q. Li, A. Z. J. Zhao, Y. Wang, K. Q. Zhang, H. T. Sun, Y. K. Lai, Int. J. Nanomedicine 2017, 12, 151.

[45] J.-W. Jeong, J. G. McCall, G. Shin, Y. Zhang, R. Al-Hasani, M. Kim, S. Li, J. Y. Sim, K.I. Jang, Y. Shi, et al., Cell 2015, 162, 662.

[46] AkzoNobel, Expancel Microspheres, n.d.

[47] AkzoNobel, n.d., https://www.akzonobel.com/expancel/system/Images/A.

[48] Y. Yang, K. Huang, G. Yang, D. Yin, Y. Zhou, Y. Qin, in Micro Nano Technol. (Ed: Y. B. T.-M. E. and T. (Second E. Qin), William Andrew Publishing, Boston, 2015, pp. 257-276.

[49] M. Gayake, D. Bodas, S. Gangal, Polymer (Guildf). 2011, 1.

[50] W. J. Hwang, K. S. Shin, J. H. Roh, D. S. Lee, S. H. Choa, Sensors 2011, 11, 2580.

[51] S. M. Khan, S. F. Shaikh, N. Qaiser, M. M. Hussain, IEEE Trans. Electron Devices 2018, 65, 5038.

[52] S. Spieth, Freidok.Uni-Freiburg.De 2013.

[53] T. Dow Chemical Company, SYLGARD ${ }^{T M} 184$ Silicone Elastomer FEATURES \&amp; BENEFITS, 2017. 
[54] Frank M White, Viscous Fluid Flow, McGraw-Hill, New York, NY, 1991.

[55] G. S. Fiorini, D. T. Chiu, Biotechniques 2005, 38, 429.

[56] J. M. Nassar, S. M. Khan, D. R. Villalva, M. M. Nour, A. S. Almuslem, M. M. Hussain, npj Flex. Electron. 2018, 2, 1.

[57] R. Nave, "Resistor Temperature Dependence," n.d.

[58] R. A. Matula, J. Phys. Chem. Ref. Data 1979, 8, 1147.

[59] M. Rajabi, N. Roxhed, R. Z. Shafagh, T. Haraldson, A. C. Fischer, W. van der Wijngaart, G. Stemme, F. Niklaus, PLoS One 2016, 11, e0166330.

[60] L. Filipovic, A. Lahlalia, J. Electrochem. Soc. 2018, 165, B862.

[61] C. K. S. Sunipa Roy, MEMS and Nanotechnology for Gas Sensors, 2015. 